\title{
Gamma Oscillations in the Hyperkinetic State Detected with Chronic Human Brain Recordings in Parkinson's Disease
}

\author{
Nicole C. Swann, ${ }^{1}$ Coralie de Hemptinne, ${ }^{1}$ Svjetlana Miocinovic, ${ }^{2}$ Salman Qasim, ${ }^{1}$ @Sarah S. Wang, ${ }^{2}$ Nathan Ziman, ${ }^{2}$ \\ Jill L. Ostrem, ${ }^{2}$ Marta San Luciano, ${ }^{2}$ Nicholas B. Galifianakis, ${ }^{2}$ and Philip A. Starr ${ }^{1,3,4}$ \\ Departments of ${ }^{1}$ Neurological Surgery and ${ }^{2}$ Neurology, ${ }^{3}$ Kavli Institute for Fundamental Neuroscience, and ${ }^{4}$ Graduate Program in Neuroscience, University \\ of California, San Francisco, California 94143
}

Hyperkinetic states are common in human movement disorders, but their neural basis remains uncertain. One such condition is dyskinesia, a serious adverse effect of medical and surgical treatment for Parkinson's disease (PD). To study this, we used a novel, totally implanted, bidirectional neural interface to obtain multisite long-term recordings. We focus our analysis on two patients with PD who experienced frequent dyskinesia and studied them both at rest and during voluntary movement. We show that dyskinesia is associated with a narrowband gamma oscillation in motor cortex between 60 and $90 \mathrm{~Hz}$, a similar, though weaker, oscillation in subthalamic nucleus, and strong phase coherence between the two. Dyskinesia-related oscillations are minimally affected by voluntary movement. When dyskinesia persists during therapeutic deep brain stimulation (DBS), the peak frequency of this signal shifts to half the stimulation frequency. These findings suggest a circuit-level mechanism for the generation of dyskinesia as well as a promising control signal for closed-loop DBS.

Key words: deep brain stimulation; dyskinesia; electrocorticography; local field potentials; motor cortex; Parkinson’s disease

Significance Statement

Oscillations in brain networks link functionally related brain areas to accomplish thought and action, but this mechanism may be altered or exaggerated by disease states. Invasive recording using implanted electrodes provides a degree of spatial and temporal resolution that is ideal for analysis of network oscillations. Here we used a novel, totally implanted, bidirectional neural interface for chronic multisite brain recordings in humans with Parkinson's disease. We characterized an oscillation between cortex and subcortical modulators that is associated with a serious adverse effect of therapy for Parkinson's disease: dyskinesia. The work shows how a perturbation in oscillatory dynamics might lead to a state of excessive movement and also suggests a possible biomarker for feedback-controlled neurostimulation to treat hyperkinetic disorders.

\section{Introduction}

Synchronized oscillatory activity is a property of healthy brain circuits, which may be dynamically modulated to coordinate long-distance communication across distributed brain networks

Received March 28, 2016; revised May 4, 2016; accepted May 7, 2016.

Author contributions: N.C.S., C.d.H., J.L.O., and P.A.S. designed research; N.C.S., C.d.H., S.M., S.Q., S.S.W., N.Z., M.S.L., N.B.G., and P.A.S. performed research; N.C.S., C.d.H., and S.M. analyzed data; N.C.S., C.d.H., S.M., S.Q., S.S.W., N.Z., J.L.O., M.S.L., N.B.G., and P.A.S. wrote the paper.

This study was supported by the University of California President's Postdoctoral Fellowship (N.C.S.), the National Institute of Neurological Disorders and Stroke (R01 NS090913-01; P.A.S.), and the Michael J. Fox Foundation (C.D.H.). We thank Dr. Alexandra Nelson for her helpful comments and critical review of this manuscript and Andrew Miller for his help with the cortical reconstructions in Figure $1 B$.

University of California, San Francisco has filed a preliminary patent application based on results from this manuscript. N.C.S., C.D.H., J.L.O., and P.A.S. are coinventors on this patent. Devices and technical support were provided by Medtronic Inc. under a research agreement.

Correspondence should be addressed to Dr. Nicole C. Swann, University of San Francisco, 513 Parnassus Health Science East 8, Room 823, San Francisco, CA 94143. E-mail: Nicole.Swann@ucsf.edu.

DOI:10.1523/JNEUROSCI.1128-16.2016

Copyright $\odot 2016$ the authors $\quad 0270-6474 / 16 / 366445-14 \$ 15.00 / 0$
(Fries, 2005; Siegel et al., 2012). However, pathological changes in oscillatory activity may contribute to brain disorders. In untreated Parkinson's disease (PD), excessive synchronized activity in the beta band $(13-30 \mathrm{~Hz})$ may constrain neural activity into an inflexible pattern, preventing dynamic changes necessary for normal movement generation (Brown and Williams, 2005; Hammond et al., 2007; Moran et al., 2008). This excessive synchronization may be responsible for some of the cardinal symptoms of PD, such as akinesia and rigidity (Kühn et al., 2006, 2008; de Hemptinne et al., 2015).

Network oscillations might also play a role in the adverse effects caused by PD therapies. One such effect is dyskinesia. Dyskinesia is characterized by involuntary choreiform movements associated with dopaminergic medication and/or deep brain stimulation (DBS; Bastide et al., 2015). These movements may preclude optimal therapeutic benefit from medication or DBS. Local field potential (LFP) recordings from cortex and basal ganglia in a rodent model of parkinsonism have shown that 
Table 1. Baseline characteristics of study patients

\begin{tabular}{llllllll}
\hline $\begin{array}{l}\text { Patient } \\
\text { no. }\end{array}$ & Gender & $\begin{array}{l}\text { Age } \\
\text { (years) }\end{array}$ & $\begin{array}{l}\text { Disease } \\
\text { duration } \\
\text { (years) }\end{array}$ & $\begin{array}{l}\text { ECoG/LFP } \\
\text { side }\end{array}$ & $\begin{array}{l}\text { Baseline UPDRS III } \\
\text { score (on/off) }\end{array}$ & $\begin{array}{l}\text { MOCA } \\
\text { score }\end{array}$ & $\begin{array}{l}\text { BDI } \\
\text { score }\end{array}$ \\
\hline 1 & $\mathrm{~F}$ & 47 & 15 & $\mathrm{~L}$ & $16 / 68$ & 28 & 14 \\
2 & $\mathrm{M}$ & 59 & 7 & $\mathrm{~L}$ & $14 / 29$ & 30 & 12 \\
$3^{a}$ & $\mathrm{M}$ & 62 & 8 & $\mathrm{R}$ & $30 / 14$ & 28 & 0 \\
$4^{a}$ & $\mathrm{M}$ & 60 & 14 & $\mathrm{R}$ & $44 / 31$ & 29 & 11 \\
\hline
\end{tabular}

MOCA, Montreal Cognitive Assessment; BDI, Beck Depression Inventory; L, left; R, right.

${ }^{a}$ Data from these patients appear only in Figure 10.

levodopa-induced dyskinesias are associated with an increase in gamma oscillatory power in both structures (Halje et al., 2012). Network changes associated with dyskinesia have not been established in humans.

Invasive human data have been obtained mainly from the basal ganglia, through temporarily externalized leads in the early postoperative period (Brown and Williams, 2005; Kühn et al., 2006, 2008; Hammond et al., 2007). Narrowband gamma oscillations have been reported in human basal ganglia recordings, but the relationship to dyskinesia and the consistency of the effect are not clear (Cassidy et al., 2002; Williams et al., 2002; Alonso-Frech et al., 2006; Alegre et al., 2012; Weinberger et al., 2012; Cagnan et al., 2014). Timing and duration of dyskinesia can be unpredictable, and they are often reduced immediately following DBS surgery, limiting the utility of short-term recording strategies.

To circumvent these shortcomings, we used a totally implantable bidirectional neural interface in humans requiring DBS implantation for the treatment of motor fluctuations and medication-induced dyskinesia. This investigational device,
Activa PC $+\mathrm{S}$ (Medtronic), has the same stimulating capabilities as standard DBS devices, but also allows long-term recording and storage of electrocorticography (ECoG) potentials and LFPs. With the view that abnormal movement in PD arises from cortical-basal ganglia interactions (Silberstein et al., 2005; de Hemptinne et al., 2013, 2015), we simultaneously recorded potentials from a permanently implanted, fourcontact ECoG strip placed over motor cortex, as well as from the therapeutic subthalamic nucleus (STN) four-contact lead. We focused our analysis on data from two patients collected over 12 months. We show that dyskinesia is associated with the emergence of a narrowband gamma oscillation throughout the basal ganglia-thalamocortical motor loop, which is modulated by DBS. This oscillatory activity suggests new strategies for feedback-controlled DBS that could limit hyperkinetic adverse effects.

\section{Materials and Methods}

Consent, regulatory approvals, and patient selection. This protocol was approved by the University of California, San Francisco institutional review board (Protocol 13-10878) under a physician-sponsored investigational device exemption (G120283). The study was registered at ClinicalTrials.gov (Identifier NCT01934296). Informed consent was obtained under the Declaration of the Principles of Helsinki. During this study, five patients with PD (two female, three male) were implanted with the Activa $\mathrm{PC}+\mathrm{S}$ at our center, but only two patients (one female, one male) had a large number of recordings with and without dyskinesia, both on and off medication and on and off DBS, as well as favorable signal-to-noise ratios at gamma band frequencies. Therefore, our statistical analysis of grouped data is restricted to these two patients. A much smaller data set is available from two additional patients, so for these, only individual examples are shown. One of these patients had only rare dyskinesia after DBS implantation, and the other had a low signal-to-
A

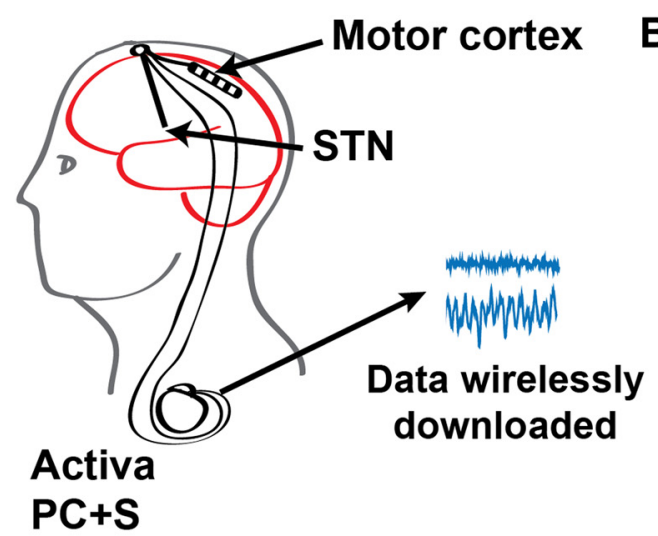

C

STN

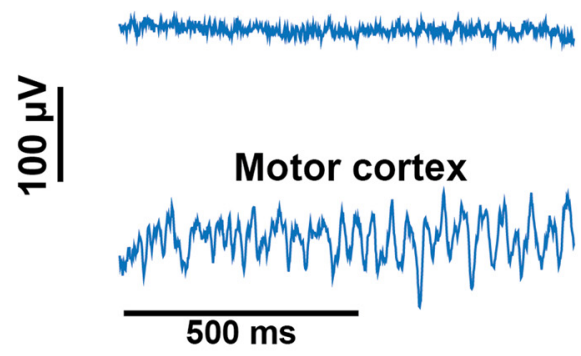

B

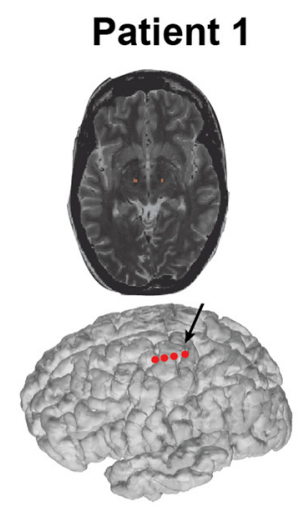

D

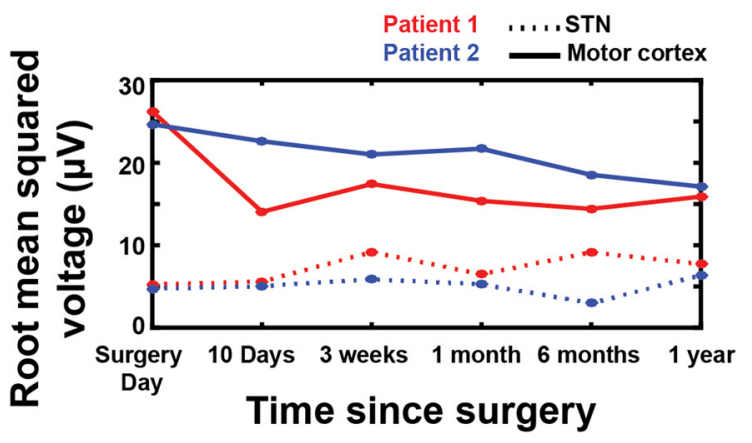

Figure 1. Electrode locations, raw signals, and signal stability over time. $\boldsymbol{A}$, Schematic of the Activa PC + S. $\boldsymbol{B}$, Electrode locations (indicated in red) for Patients 1 and 2 over cortex (top) and in STN (bottom). Locations are derived from the preoperative MRI merged with the intraoperative CT. C, Raw signals from STN and Motor cortex. D, Root mean square voltage off DBS for motor cortex ECoG potentials and STN LFP for each patient, recorded over 12 months. Signals are relatively stable over time. 

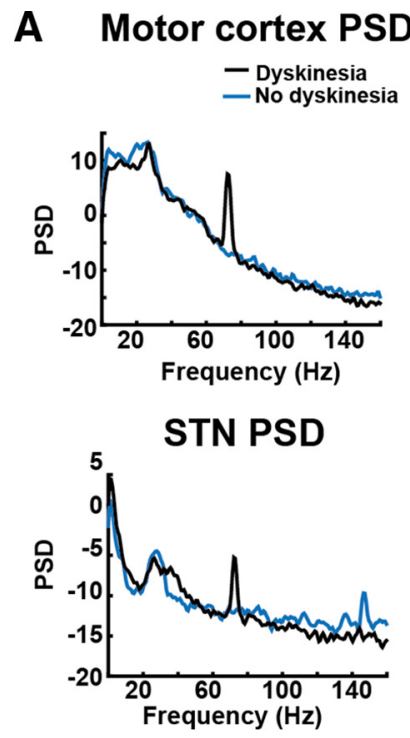

C

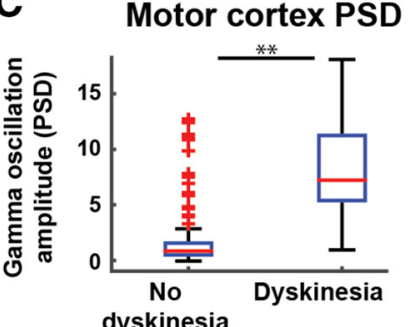

STN PSD

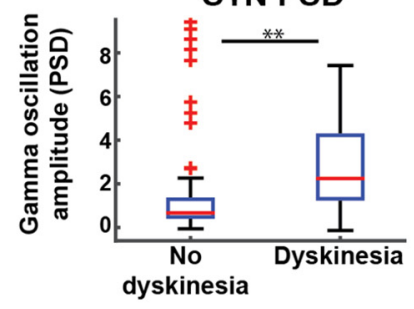

Coherence

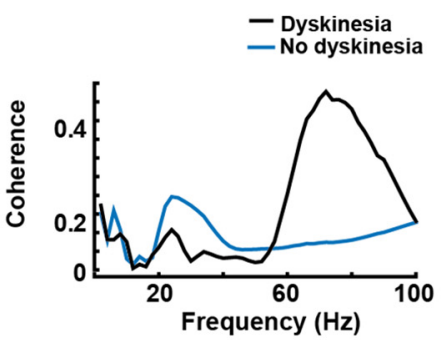

Phase coherence

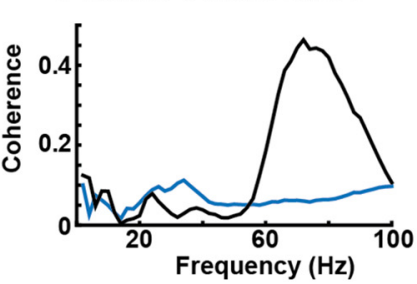

Coherence

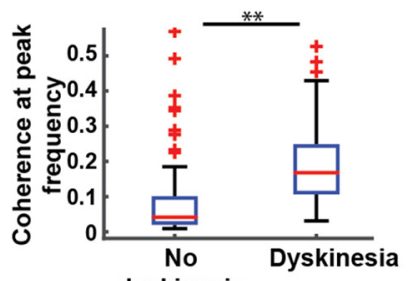

dyskinesia

Phase coherence

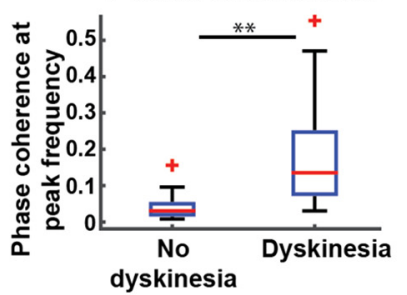

B 1. Original PSD

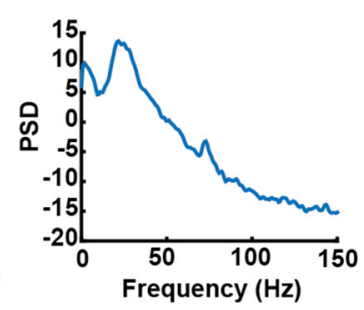

3. Calculation of PSD height

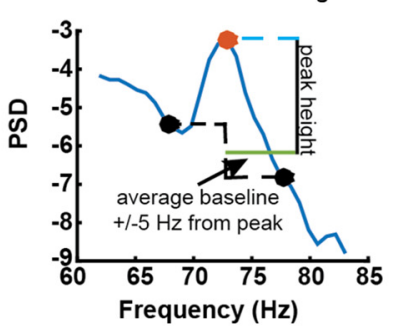

Motor cortex PSD

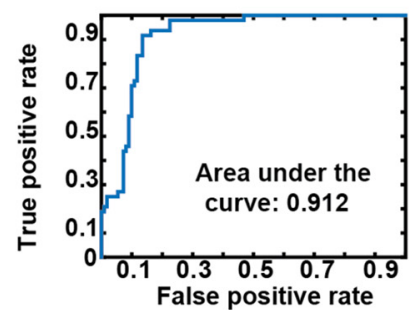

STN PSD

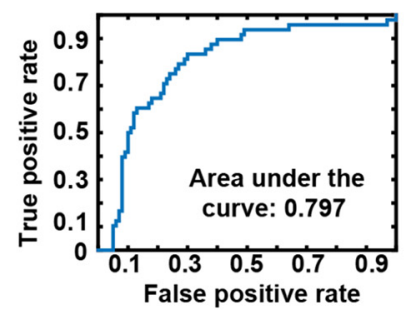

2. Flattened and normalized PSD to find peak frequency in $62-83 \mathrm{~Hz}$ range

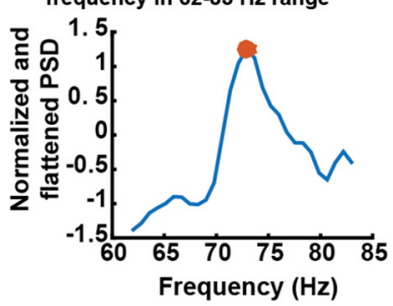

4. Calculation of PSD width at half the height

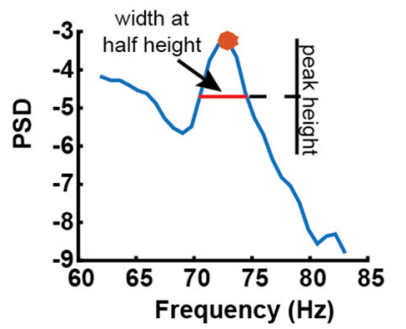

Coherence

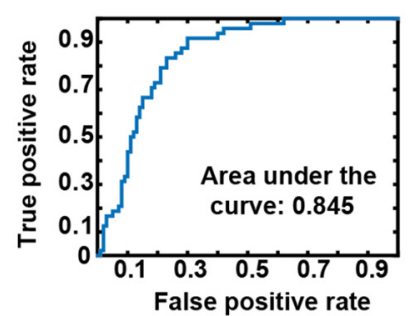

Phase Coherence

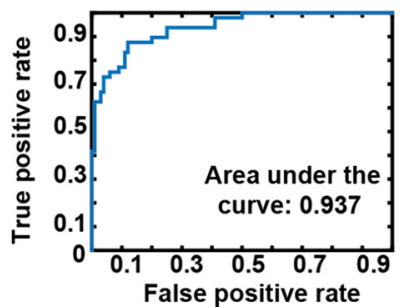

Figure 2. Gamma oscillations distinguish the dyskinetic and nondyskinetic states. $A$, Example of motor cortex log PSD, STN log PSD, coherence, and phase coherence from Patient 2 at rest (all on medications). The PSD scale is $10{ }^{*} \log _{10}\left(\mu \mathrm{V}^{2} / \mathrm{Hz}\right.$ ). B, Schematic of PSD height and width calculation. Original PSDs (panel 1) were flattened and normalized (panel 2; see Materials and Methods) to identify the peak frequency in the $62-83 \mathrm{~Hz}$ range (red dot). The height of this peak was calculated by subtracting the original log PSD value at this frequency (red dot) from the average of the PSDs $5 \mathrm{~Hz}$ above and below the peak frequency (black dots, average indicated with green line). The height is indicated with the vertical black line (panel 3). The width of the peak at half the height was also calculated (red line, panel 4). For coherence, the maximum coherence in the $62-83 \mathrm{~Hz}$ range was used as the height. C, Group analysis for peak gamma amplitude. There was significantly higher gamma power during dyskinesia for each measure (Table 2). Data are displayed as boxplots, where center is median, box top and bottom are 25th and 75th percentiles, and whiskers reflect range of data excluding outliers. Outliers are denoted with plus signs. ${ }^{* *}$ indicates $p<0.001$. D, Receiver operating characteristic curves for each measure showing the false positive and true positive rates derived from fitting the data to a general linear model and examining rates for different classification threshold values for each biomarker.

noise ratio for cortical recordings such that frequencies $>70 \mathrm{~Hz}$ were near or below the noise floor of the device and not reliably detected. The final patient did not experience dyskinesia after implantation, so no data from that subject were included. Baseline characteristics of the two patients included in the statistical analysis as well as the two additional patients for whom we present example data are provided in Table 1.

Clinical characterization. Study patients were evaluated by a movement disorders neurologist and met criteria for a diagnosis of PD (i.e., presence of bradykinesia and at least one other parkinsonian cardinal sign and responsiveness to levodopa). Baseline motor function in the on and off medication states were characterized using the Unified Parkinson's Disease Rating Scale, motor subscale (UPDRS III). Patients were evaluated by a neuropsychologist to exclude significant cognitive impairment or untreated mood disorder.

Surgery. A quadripolar subthalamic lead (model 3389, Medtronic) was placed using frame-based stereotaxy and confirmed by microelectrode recording in the awake state using standard methods (Starr et al., 2002). Proper location in the motor territory of the STN was verified by eliciting movement-related single-cell discharge patterns (Starr et al., 2002). A quadripolar cortical ECoG lead was placed in the subdural space through the same burr hole used for the subthalamic lead. For Patient 1, we used a cylindrical lead (model 3391, Medtronic) with $3 \mathrm{~mm}$ contact length and $4 \mathrm{~mm}$ intercontact spacing; for all other patients, we used a flat lead (model 3587A25, Medtronic), with $4 \mathrm{~mm}$ contact diameter and $10 \mathrm{~mm}$ intercontact spacing. At least one contact covered the posterior precentral gyrus (presumed primary motor cortex), $\sim 3 \mathrm{~cm}$ from the midline on the medial aspect of the hand knob (Yousry et al., 1997). Adequate localization of the ECoG strip was confirmed using intraoperative CT merged to the patient's preoperative MRI, as described previously (Shahlaie et al., 2011). Functional localization of the ECoG strip was verified with somatosensory-evoked potentials, as reported previously (Crowell et al., 2012). If time permitted, a movement task described previously (de Hemptinne et al., 2015) was also performed to ensure that canon- 


\section{A Patient 1}
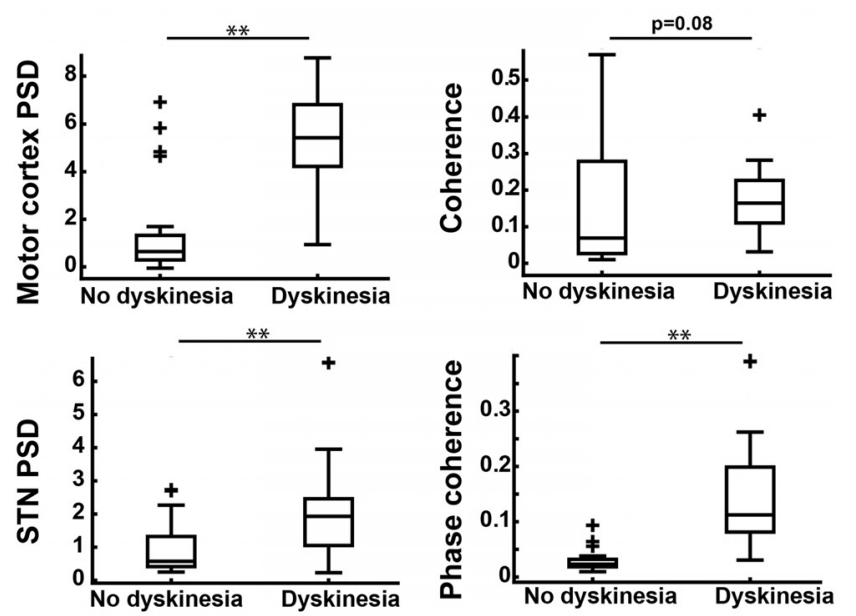

B Patient 2
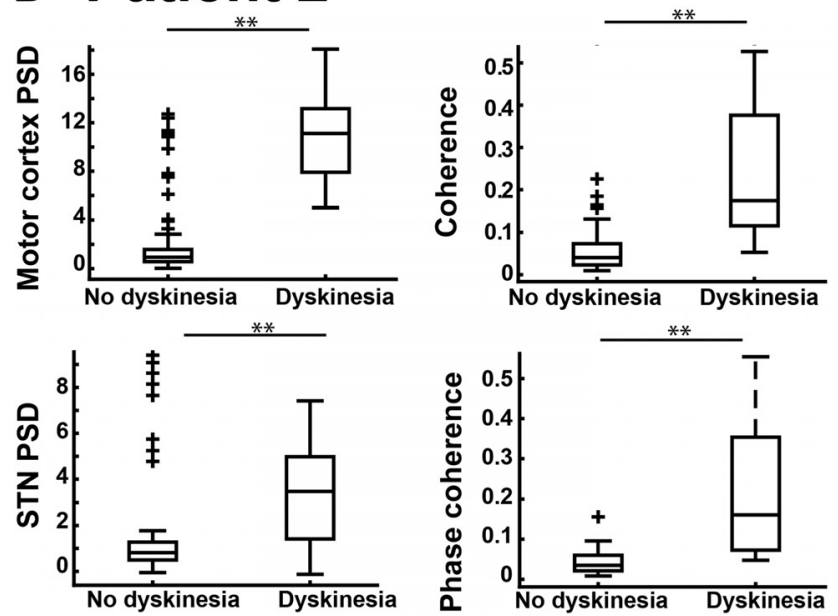

Figure 3. $\quad \boldsymbol{A}, \boldsymbol{B}$, Grouped data from Figure 2 (segregated by patient (Patient $1, \boldsymbol{A} ;$ Patient $2, \boldsymbol{B}$ ) for each measure examined (motor cortex and STN PSD, coherence, and phase coherence). $p$ values are reported in Table 2 . Data are displayed with boxplots as in Figure $2 C .{ }^{* *}$ indicates $p<0.001$.

ical movement-related beta decreases and broadband gamma increases were produced (Crone et al., 1998a; Crone et al., 1998b). The exiting wire from the cortical contact array was secured to the skull with a titanium miniplate. The free ends of the cortical and subthalamic leads were coiled under the ipsilateral parietal scalp.

The remaining hardware was placed under general anesthesia. In the posterior parietal area, the free ends of the cortical and subthalamic leads were connected to $40 \mathrm{~cm}$ lead extenders (model 37087, Medtronic), which were tunneled down the neck to a Medtronic Activa $\mathrm{PC}+\mathrm{S}$ placed in a pocket over the pectoralis muscle (Fig. $1 \mathrm{~A}$ ). The Activa $\mathrm{PC}+\mathrm{S}$ is identical in shape and size to the standard Activa PC. For all patients except Patient 2, a contralateral STN electrode was implanted and attached to a separate Activa SC pulse generator, for clinical purposes only, so that bilateral therapy could be delivered. Patient 2 only received unilateral therapy, as clinically indicated.

Experimental design. Following surgery, each patient participated in research visits at regular intervals during which ECoG potentials and STN LFPs were recorded and downloaded wirelessly. These visits included recordings on and off medication in the month before initial DBS programming, followed by visits on and off of therapeutic DBS. One month after implantation, programming of the STN lead(s) was performed to achieve the best clinical result. The cortical lead was not used for stimulation.
During formal study visits, STN and cortical field potentials were recorded at rest, during an iPad reaching task that has been described previously (de Hemptinne et al., 2015), and during walking. Rest and walking recordings were 1 to $2 \mathrm{~min}$ long, and iPad reaching task recordings were $3-5 \mathrm{~min}$ long. Some additional brain recordings were initiated in our clinic to document specific phenomena, such as briefly shifting DBS frequency during an episode of dyskinesia to document a shift in the gamma peak frequency (described in Results).

Patients were also given a home data-collection triggering device (Intercept Patient Programmer, model 37441), allowing the patient to initiate brain recordings at home for a prespecified duration (1 min). Patients were instructed to initiate such recordings if they were experiencing dyskinesia, particularly severe "off" periods, or if they were feeling especially asymptomatic. They were also instructed to take notes on how they felt during each recording, detailing any notable symptoms.

Recordings. Investigator-initiated recordings were activated via the Sensing Programmer (model 8181) and Sensing Programmer software (model 8180), which are part of the Medtronic Activa PC + S system. The Activa $\mathrm{PC}+\mathrm{S}$ device allows a maximum of two time domain channels (one from each lead) to be recorded simultaneously. Thus, we typically recorded from one bipolar contact pair in motor cortex and one from the STN, with a sampling rate of $800 \mathrm{~Hz}$, unless otherwise specified. To capture data as rapidly as possible from multiple cortical electrodes, we occasionally performed brief "montage recordings," during which the device sampled data from each cortical electrode pair sequentially. Data from each electrode pair were recorded for $30 \mathrm{~s}$, before proceeding to the next pair. For these recordings, a sampling rate of $422 \mathrm{~Hz}$ was used.

In general, the cortical contact pair used for recordings was selected based on which pair showed the clearest somatosensory evoked potential, the strongest movement-related broadband gamma response, and/or strongest beta peak at rest, at the time of initial surgical insertion. Typically, there was good overlap for these three measures in terms of identifying one or two contact pairs. When ambiguity remained, the preoperative MRI merged to the postoperative CT was used to help select an optimal motor cortex contact pair. Additional contacts were sometimes used, particularly if more than one contact pair had shown strong movement-related signals as described above.

The selection criteria for the STN recording configuration differed before and after therapeutic DBS was activated at 1 month postimplantation, since optimal recording contact pairs depended on which contacts were used for stimulation [stimulation and recording could not be performed from the same contact(s) simultaneously]. Stimulation contacts were not known before the patient's initial DBS programming. Before stimulation, recording contacts were based on which contact pair had the largest beta peak the day after surgery (off PD medications). If selection was ambiguous, the center contacts ( 1 and 2 ) were used. After stimulation, recording contacts bordering stimulation contacts on either side were used to minimize artifact. Occasionally, no STN contacts were recorded either because an unusually long cortical recording was desired or more recordings than usual were performed, precluding recording from two sites due to the limited Activa $\mathrm{PC}+\mathrm{S}$ memory storage capacity.

The Activa $\mathrm{PC}+\mathrm{S}$ has several built-in filters. We avoided filters when possible, so our initial recordings used minimal filters $(0.5 \mathrm{~Hz}$ high-pass filter and a built in $260 \mathrm{~Hz}$ antialiasing filter) and maximum gain (2000). However, after our early patient initiated therapeutic stimulation, we realized that saturation of the amplifier was sometimes occurring, so we began using a $100 \mathrm{~Hz}$ low pass filter, and, in cases of especially large stimulation artifact, gain was reduced to 1000 for the STN. The Activa $\mathrm{PC}+\mathrm{S}$ also has a data compression feature that we did not use, to avoid compression-associated reduction in the signal-to-noise ratio.

Data analysis. Analyses were performed in Matlab using a combination of built-in Matlab functions, EEGlab functions (Delorme and Makeig, 2004), and custom functions/scripts. Power spectral density (PSD) calculations used the Welch method ("pwelch" in Matlab, with a window length of $512 \mathrm{~ms}$ and a FFT length of 1024). The log of the PSD was then taken. To calculate coherence, we first filtered both the ECoG and the LFP signals at frequencies ranging from 2 to $50 \mathrm{~Hz}$, with a $2 \mathrm{~Hz}$ bandwidth using a two-way finite impulse response 1 (FIR1) filter ("eegfilt" in EEGlab). Complex signals were then obtained for each filtered 
Off DBS
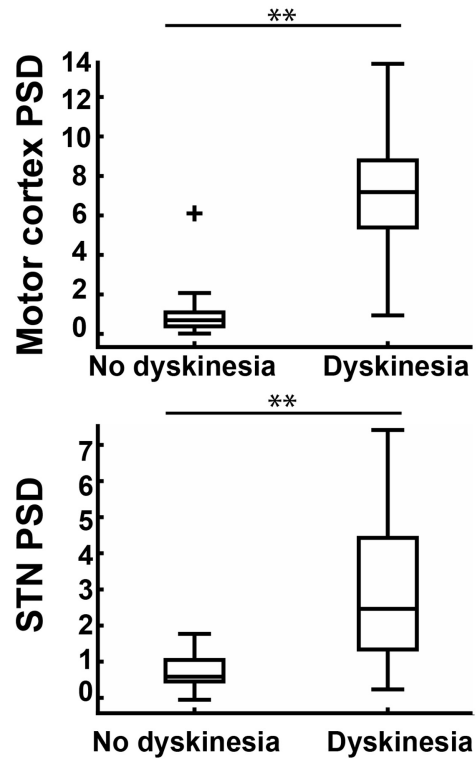

Figure 4. Grouped data from Figure $2 \mathrm{C}$ for recording off DBS only. Values are significantly higher during dyskinesia for all measures, demonstrating that our findings are not driven by a stimulation artifact. $p$ values reported in Table 3. Data are displayed with boxplots as in Figure $2 C .{ }^{* *}$ indicates $p<0.001$.

signal by taking the Hilbert transform of the filtered signal. Coherence was calculated as the cross-spectrum of the two signals, normalized by each signal's autospectrum. For phase coherence, a similar procedure was used, but instead of considering the entire complex signal, only the phase was extracted from each filtered signal using a Hilbert transform. Then the instantaneous phase difference between the two signals was taken (accounting for the fact that phase is a circular signal). Plots that show the instantaneous phase differences are derived from these phase difference values (i.e., instantaneous phase differences for the entire signal). To obtain phase coherence, the absolute value of the average of the instantaneous phase differences was calculated (i.e., the vector length, which signifies the consistency of the phase difference between regions).

Categorization of files for group analysis. For each patient, we separated files into groups with and without dyskinesia based on the dyskinesia rating scales (Unified Dyskinesia Rating Scale Part III; Goetz et al., 2008) obtained just before or just after the relevant data recording session by a movement disorder neurologist, who did not analyze the electrophysiological data. If the dyskinesia rating scale score was greater than zero for the contralateral arm or trunk, the file was considered "with dyskinesia," and if ratings were zero, the file was considered "without dyskinesia." To ensure correct categorization, we also evaluated video collected during the data recordings (acquired during many, but not all, researcherinitiated data recordings) to confirm the presence or absence of dyskinesia. Recordings during rest, the iPad reaching task, and walking were included in the analysis. Additionally, for home recordings, if the patients noted that they were experiencing either "dyskinesia" or "involuntary movement" that was not tremor, the file was considered "with dyskinesia," and if the patient's notes did not indicate dyskinesia, it was considered "without dyskinesia."

Recordings where the DBS settings were changed during the recording (wash-in or washout or a change in DBS parameters) were excluded from the group analysis. Likewise, recordings from cortical contact pairs that never showed the narrowband gamma oscillation described in Results (regardless of dyskinesia status) were presumed to be insensitive detectors and were excluded. (However, we conducted a control analysis including these recordings and results did not differ.) Additionally, home recordings where the patient did not take notes or where notes were not clearly associated with a specific recording were excluded. Finally "montage recordings," where data were sampled at $422 \mathrm{~Hz}$ (instead of $800 \mathrm{~Hz}$ ), were excluded.
Measurement of gamma oscillation parameters. For each recording, the maximum cortical or STN PSD peak between $62-83 \mathrm{~Hz}$ was extracted, and a peak "height" was calculating by subtracting the log PSD from the average log PSDs $5 \mathrm{~Hz}$ above and below the frequency of the peak. [To determine the frequency of the peak, a normalization procedure was performed. First, the spectrum was approximately flattened by fitting it to a fourth-order polynomial, excluding artifacts, to correct for the $1 / f$ pattern (Pritchard, 1992). Then, the entire spectrum was normalized by subtracting the mean and dividing by the standard deviation across all frequencies. This method was used only to identify the frequency of the peak. The height of the peak at this frequency was calculated based on the original PSD as described above.] The method of quantifying gamma peak height is illustrated in Figure $2 B$. The width of the peak at half the height was also calculated.

Coherence and phase coherence did not require a correction for the $1 / f$ decrease because coherence values did not exhibit the strong $1 / f$ decrease in amplitude present in the PSD (Fig. 2A). Additionally, because coherence is internally normalized, raw coherence values were used for the statistical analyses rather than first calculating the peak height relative to neighboring frequencies.

Measurement of beta oscillation parameters. Due to the importance of beta band synchronization in the motor system and in PD in particular (Crone et al., 1998b; Hammond et al., 2007), we also examined our four measures of interest (motor cortex PSD, STN PSD, coherence, and phase coherence) in the beta frequency range $(13-30 \mathrm{~Hz})$. Log PSD values between 13 and $30 \mathrm{~Hz}$ were averaged to derive the PSD measures. Coherence values were derived in the same manner as described for the gamma oscillation analysis. Note that because of DBS artifacts in the beta range (at folded subharmonics), we included only recordings made in the absence of stimulation for this analysis.

Statistics. The values for all four variables of interest (motor cortex PSD, STN PSD, coherence, and phase coherence) were compared between groups (dyskinesia vs no dyskinesia) using a two-tailed, nonparametric Wilcoxon rank sum test. We tested this for each of the two patients separately (Fig. 3) as well grouped together (Fig. 2C). To determine whether the stimulation artifact may be driving any results, we also ran the same analyzes including only recordings with DBS off (Fig. 4). To determine how well the height of the gamma PSD peak or the coherence values would work as classifiers for dyskinesia and to characterize the specificity and sensitivity of the biomarkers, we derived receiver operating characteristic (ROC) curves (Zwieg and Campbell, 1993). These were derived using the "perfcurve" function in Matlab (Fig. 2D). Values were first fit to a logistical regression model ("fitglm") using a binomial distribution.

\section{Results}

Recording locations, signal characteristics, and data overview Recording sites in cortex and basal ganglia and example recordings from each site are shown in Figure 1, $B$ and $C$. Average root mean square voltage for recordings with DBS off, were $6.1 \mu \mathrm{V}$ for STN and $19 \mu \mathrm{V}$ for motor cortex. Signal amplitudes for recordings from both cortex and STN were stable for the 12 months of the study (Fig. 1D). The number of recordings used for group analyses are reported in Table 2. For each recording, the longest artifact-free segment of data was used for analysis. The mean duration of recordings (excluding artifacts) was $96.5 \mathrm{~s}$ (SD, $72 \mathrm{~s}$; range, $28-302 \mathrm{~s}$ ). There was no 
Table 2. p values and statistical parameters during dyskinetic vs nondyskinetic states (on and off DBS)

\begin{tabular}{|c|c|c|c|c|c|c|}
\hline Measure & $\begin{array}{l}\text { Total number } \\
\text { of recordings } \\
\text { (Patient 1/Patient 2) }\end{array}$ & $\begin{array}{l}\text { Number of recordings } \\
\text { with dyskinesia total } \\
\text { (Patient 1/Patient 2) }\end{array}$ & $p$ values total & $\begin{array}{l}p \text { values individual patients } \\
\text { (Patient 1/Patient 2) }\end{array}$ & ROCAUC & $\begin{array}{l}\text { True positive/false } \\
\text { positive for optimal } \\
\text { point }\end{array}$ \\
\hline Motor cortex PSD & $159(52 / 107)$ & $48(23 / 25)$ & $2.22 \times 10^{-16}$ & $3.024 \times 10^{-7} / 2.48 \times 10^{-11}$ & 0.9123 & $0.9167 / 0.1351$ \\
\hline STN PSD & $148(52 / 96)$ & $48(23 / 25)$ & $5.122 \times 10^{-9}$ & $6.99 \times 10^{-4} / 1.5 \times 10^{-6}$ & 0.7973 & $0.5833 / 0.1200$ \\
\hline Coherence & $148(52 / 96)$ & $48(23 / 25)$ & $1.16 \times 10^{-11}$ & $0.08 / 4.72 \times 10^{-10}$ & 0.8452 & $0.667 / 0.1500$ \\
\hline Phase Coherence & $148(52 / 96)$ & $48(23 / 25)$ & $<1 \times 10^{-15}$ & $1.55 \times 10^{-8} / 8.30 \times 10^{-11}$ & 0.9371 & $0.7292 / 0.0400$ \\
\hline
\end{tabular}

AUC, Area under the curve.

difference in the recording length for recordings with and without dyskinesia $(p>0.88)$. One-hundred seven of the recordings were initiated by study personnel during formal study visits at regular intervals, while fifty-two were initiated at home by the patients. Dyskinesia occurred almost exclusively in the on medication state, but could be present on or off of DBS.

Gamma oscillations in cortex and STN are associated with dyskinesia

Visual inspection of PSD plots for each cortical recording revealed that many of those collected during episodes of dyskinesia showed a discrete, narrowband peak (local maximum at peak frequencies between 62 and $83 \mathrm{~Hz}$ ). A similar but smaller peak was often present in the STN, which had strong coherence with motor cortex (both magnitude and phase) in the same frequency range (Fig. $2 A$ ). To characterize the relationship of these oscillatory phenomena to the presence of dyskinesia, we quantified the oscillation (Fig. 2B; see Materials and Methods) and segregated its amplitude by dyskinesia status. Results are presented in Figure 2C, with statistical details in Table 2. All four putative biomarkers (motor cortex and STN PSD, coherence, and phase coherence for the local maximum between 62 and $83 \mathrm{~Hz}$ ) distinguished the dyskinetic state from the nondyskinetic state, with the strongest distinction for cortical PSD and cortex-STN phase coherence. Separation of biomarker amplitudes by dyskinesia status was also present when data from each patient were analyzed independently, with the sole exception of coherence in Patient 1 (which was at the trend level, $p=0.08$; Fig. $3 A$, Table 2), showing the effects were not driven by a single patient. Separation of biomarker amplitudes also persisted when data were restricted to those with DBS off (Fig. 4, Table 3), demonstrating that the effects were not driven by stimulation artifacts, which could have interfered with accurate peak height analysis in the gamma frequency range.

ROC curves are provided for each measure in Figure $2 D$, and the area under the curve and sensitivities and specificities for detection of the dyskinetic state are listed in Table 2. Consistent with the statistical evaluation above, the gamma oscillation amplitude in motor cortex and gamma phase coherence between motor cortex and STN were better classifiers for the
Table 3. $p$ values and statistical parameters for gamma oscillation during dyskinetic versus nondyskinetic states (data from off DBS only)

\begin{tabular}{llll}
\hline Measure & $\begin{array}{l}\text { Total number } \\
\text { of recordings }\end{array}$ & $\begin{array}{l}\text { File number } \\
\text { with dyskinesia }\end{array}$ & $p$ value total \\
\hline Motor cortex PSD & 82 & 25 & $5.59 \times 10^{-12}$ \\
STN PSD & 80 & 25 & $2.97 \times 10^{-8}$ \\
Coherence & 80 & 25 & $2.86 \times 10^{-11}$ \\
Phase Coherence & 80 & 25 & $2.31 \times 10^{-11}$ \\
\hline
\end{tabular}
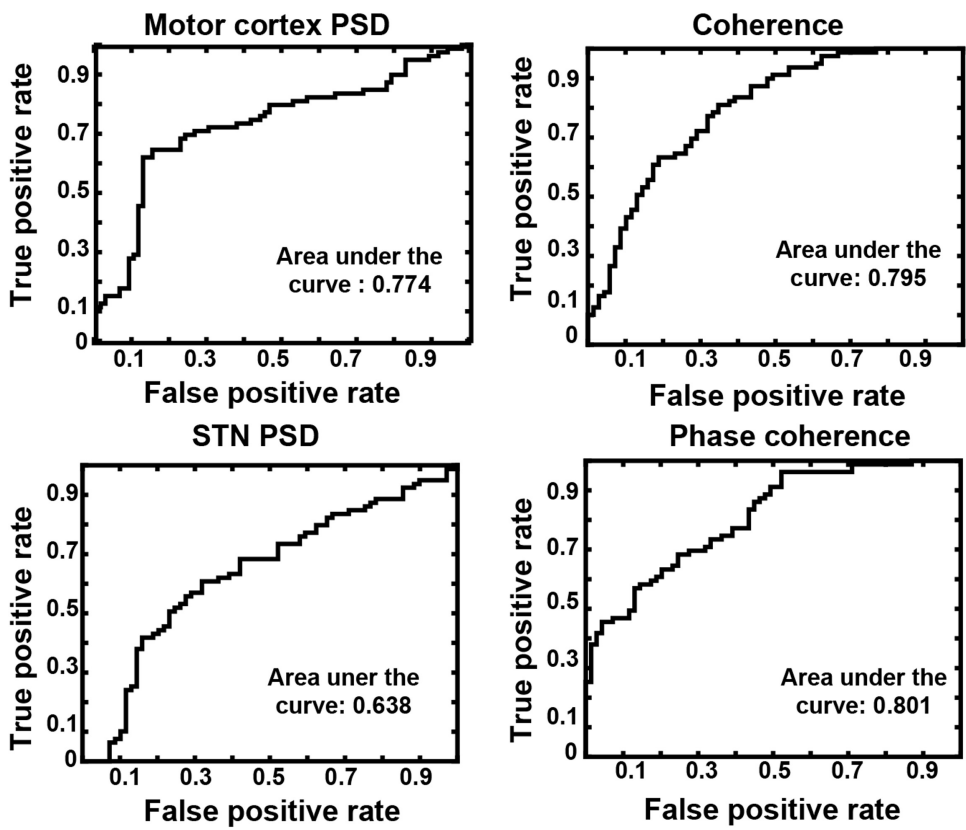

Figure 5. The gamma oscillation as a function of medication state. The oscillation is a relatively poor marker for medication state. The figure is similar to Figure $2 D$, except here recordings are segregated based on medication state rather than presence or absence of dyskinesia. All home recordings were considered "on medication" unless patients indicated that they were off medications overnight, since patients were maintaining their regular medication regimen.

presence or absence of dyskinesia than the gamma oscillation amplitude in STN or coherence between motor cortex and STN. Since dyskinesia occurred almost exclusively in the "on medication state," it is important to dissociate a medication effect from a dyskinesia effect. To show that the oscillatory biomarkers are more closely related to the dyskinetic state than the dopaminergic state (on vs off of dopaminergic medications), we also performed an ROC analysis for medication state (Fig. 5). This analysis included the same data shown in Figure $2 D$, but grouped files according to medication status. For recordings in-clinic, "off medication" recordings were after withholding medications for at least $12 \mathrm{~h}$. Recordings that the patients initiated at home were considered "on medication" unless patients indicated that they had been off medications overnight, since patients were maintaining their regular 


\section{A All recordings - distribution of phase angle differences Dyskinesia No dyskinesia}
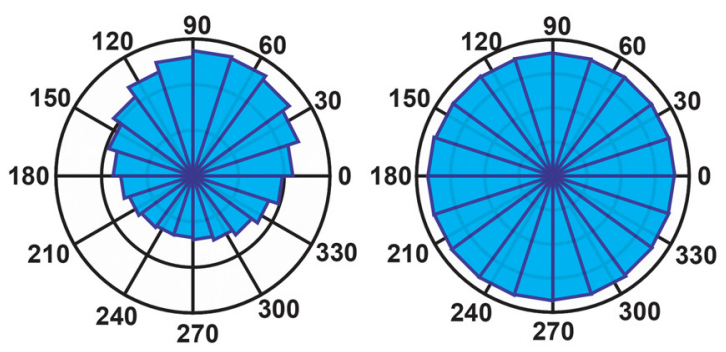

B Only DBS off - distribution of phase angle differences
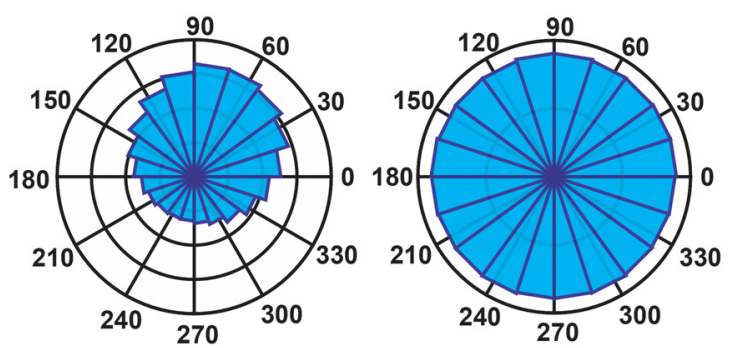

Figure 6. STN-motor cortex phase differences at the narrowband gamma frequency. $\boldsymbol{A}$, Instantaneous phase differences between STN and motor cortex grouped for all recordings (on and off DBS) with and without dyskinesia. The number of sample points per bin is different for each plot due to differing amounts of data included for each. For the "dyskinesia" recordings, the outermost marker indicates 300,000 sample points, and the second outermost indicates 200,000 . For the "no dyskinesia" recordings, the outermost marker indicates 400,000 sample points, and the second outermost indicates 300,000 . B, Instantaneous phase differences between STN and motor cortex grouped for all recordings offDBS, with and without dyskinesia. For the "dyskinesia" recordings, the outermost marker indicates 200,000 sample points, and the second outermost indicates 150,000 . For the "no dyskinesia" recordings, the outermost marker indicates 250,000 sample points, and the second outermost indicates 125,000 .

medication regimen. This analysis showed that the area under the curve for the ROC analysis derived by medication status is lower for all four gamma oscillation derived biomarkers, than when using the biomarkers to predict the presence or absence of dyskinesia.

The fact that phase coherence distinguished the two clinical states to a greater degree than coherence suggests that the hyperkinetic state is more closely related to the phase relationship between motor cortex and basal ganglia gamma oscillations than to amplitude correlations between them. To visualize this effect, we pooled the instantaneous phase angles between motor cortex and STN for all time points, all recordings, and both patients. We showed a consistent phase difference during dyskinesia, but not without dyskinesia (Fig. $6 \mathrm{~A}$ ). The mean phase angle during dyskinesia was $71^{\circ}$. This phase relationship in the dyskinetic state persisted regardless of stimulation status (on, or off; Fig. $6 B$ ), demonstrating that the presence of stimulation artifact in those files recorded with stimulation on was not responsible for the observed consistency of the phase relationship between cortex and STN.

We also examined whether the height of the narrowband gamma oscillation correlated with dyskinesia severity. While we did observe a correlation $\left(r=0.63, p=3.58 \times 10^{-13}\right.$; Fig. 7), this effect was driven most strongly by presence versus absence of dyskinesia, rather than by its severity. This is consistent with the observation in rodents that the relationship

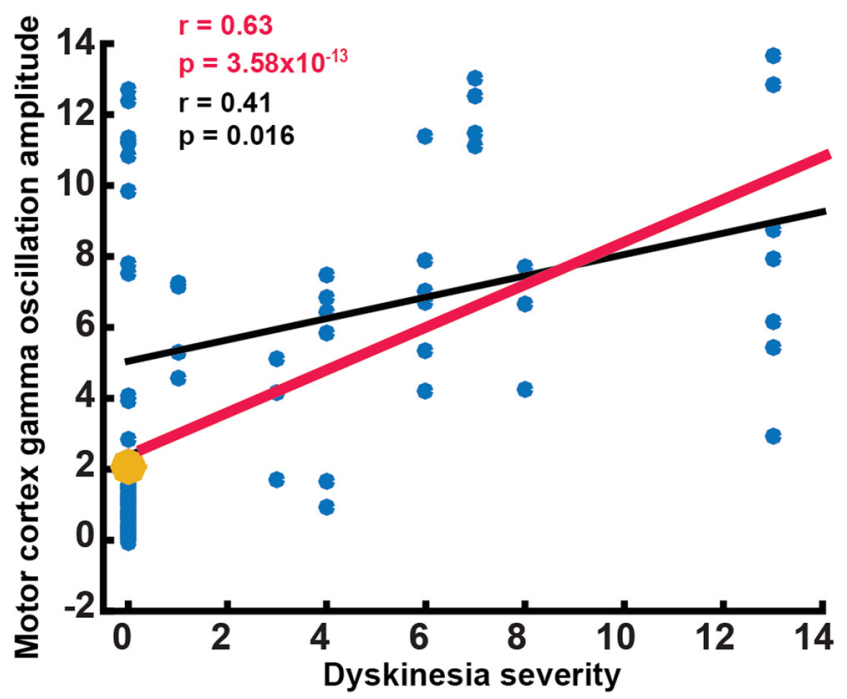

Figure 7. Correlation between dyskinesia severity and gamma oscillation amplitude. There is a correlation between dyskinesia severity (measured with dyskinesia rating scale; see Materials and Methods) and peak height of gamma oscillation in motor cortex $(r=0.63, p=3.58 \times$ $10^{-13}$, Spearman correlation, indicated with red regression line). However, this effect was mostly driven by the presence or absence of dyskinesia (when recordings with no dyskinesia were excluded, $r=0.41, p=0.016$, Spearman correlation, indicated with black regression line). This observation is consistent with a bimodal or sigmoidal relationship between dyskinesia severity and gamma oscillation height (Halje et al., 2012). Only in-clinic recordings were included in this analysis (where the dyskinesia rating scale was available, 107 recordings total). The sum of dyskinesia severity from all effectors measured with the dyskinesia rating scale (arms, legs, trunk, neck, and face) was derived to quantify dyskinesia severity. Average value for recordings without dyskinesia is indicated with the large yellow circle.

between gamma power and dyskinesia severity is sigmoidal (Halje et al., 2012).

\section{Beta oscillations in cortex and STN and association} with dyskinesia

In contrast to the gamma oscillation, in the beta range, neither motor cortex PSD, STN PSD, nor coherence distinguished the dyskinetic from the nondyskinetic states (Fig. 8). There was a significant difference in phase coherence $\left(p=7.24 \times 10^{-4}\right)$, with lower beta phase coherence between STN and motor cortex when dyskinesia was present compared to when it was absent (the opposite pattern that we observed for the gamma oscillation.) Of note we also repeated the beta range analysis with recordings categorized by medication (as performed in Fig. 5). For this analysis we also found a significant difference in phase coherence $(p=0.012)$ with lower phase coherence between motor cortex and STN when patients were on medications compared to off. We also observed lower STN PSD when patients were on medications compared to off ( $p=0.0356)$, as has been observed in previous studies (Priori et al., 2004; Kühn et al., 2006).

\section{Relationship between gamma oscillation characteristics in} cortex vs STN during dyskinesia

The relationship between gamma oscillatory characteristics in cortex versus STN, for DBS off recordings, is described in Table 4. Peaks were of greater amplitude in cortex compared to STN. The peak frequency varied between recordings, but their distributions in the two patients studied overlapped (Fig. 9A). Of note, the exact frequency of the gamma peak may be related to the time of recording relative to levodopa dose (Halje et al., 2012). There was a significant correlation (Pearson's $r=0.597, p=0.0016$ ) between the frequency of the gamma peak in cortex and STN (Fig. 
$9 B)$. Although only two subjects in the study (1 and 2) had a sufficient number of recordings in the dyskinetic state for statistical analyses (explained further in methods), very similar frequency and amplitude characteristics of the dyskinesiaassociated cortical PSD peak were seen in two other subjects who had a small number of high signal-to-noise ratio recordings in the dyskinetic state (Fig. 10). The fifth study subject had no dyskinesia after DBS implantation and did not have a narrowband gamma oscillation in cortex or STN.

\section{The gamma oscillation and voluntary movement}

To be of optimal utility as a driver of closed loop deep brain stimulation, a biomarker of abnormal movement should not be strongly affected by normal movement. To assess the effect of voluntary movement on gamma oscillation derived biomarkers, we conducted an analysis similar to that in Figure 2B, but separated recordings that included voluntary movement [walking or performing an arm movement (iPad) task] from those with-

out voluntary movement (Fig. 11). In the dyskinetic state there were no significant differences in the gamma oscillation amplitude for any measure between recordings obtained with and without voluntary movement. In contrast, the difference in the gamma oscillation for all comparisons with and without dyskinesia was highly significant.

In sensorimotor cortex, a broadband power increase is known to be associated with movement (Crone et al., 1998a; Miller et al., 2007); however, this gamma increase is over a much broader frequency range (usually $50-250 \mathrm{~Hz}$ ) and likely has a different etiology than the narrowband peak we see here (Manning et al., 2009). Additionally, the movement-related broadband power change is small in amplitude compared to the height of the peak associated with dyskinesia (Fig. 12). This point is developed further in Discussion.

\section{Localization of cortical gamma oscillation}

Precise localization of cortical contact(s) over the region of the strongest gamma oscillatory signal will be important for clinical use of a cortical detector in feedback controlled DBS. In Patient 2, we recorded files from all possible cortical contact pairs (montage recordings, see Materials and Methods) during dyskinesia and observed that the dyskinesia-associated narrowband gamma power increase was spatially specific to one contact common to all bipolar pairs showing a strong gamma oscillation (contact 11, anterior commissure-posterior commissure coordinates: $-38.02,-6.59,64.21)$. This contact localized to the anterior part of precentral gyrus, extending over the precentral sulcus (Fig. 13).

\section{STN stimulation entrains cortical gamma at half the} stimulation frequency

STN stimulation is known to be able to suppress or exacerbate dyskinesia in $\mathrm{PD}$, depending on exact stimulation parameters and contact locations (Zheng et al., 2010; Oyama et al., 2012). Both Patients 1 and 2 often experienced dyskinesia both on and off stimulation. When dyskinesia occurred in the on-stimulation state, the cortical gamma peak always occurred at half the stimulation frequency (Fig. 14A). This is unlikely to be due to stimulation artifact because it was not present when stimulation was delivered at the same settings, but in the absence of dyskinesia (Fig. 14B). Moreover, the distribution of phase angles between STN and motor cortex in the dyskinetic state, at half stimulation frequency, were similar for stimulation-on recordings compared to stimulation-off recordings. In contrast, a more narrow (presumed artifactual) phase angle distribution is observed at the actual frequency of stimulation and at folded subharmonics of stimulation frequency (Fig. 14D). These findings are consistent with reports of "partial entrainment" of neuronal discharge by STN DBS at therapeutic frequencies (Garcia et al., 2003; Hashimoto et al., 2003; Li et al., 2012; Agnesi et al., 2015). Our findings suggest that when this entrainment occurs in the frequency range associated with dyskinesia, DBS cannot suppress the dyskinetic state. As a proof of principle, we showed that the frequency at which the peak occurred could be moved by changing the stimulation frequency, in this example from $65 \mathrm{~Hz}$ with $130 \mathrm{~Hz}$ stimulation to $75 \mathrm{~Hz}$ with $150 \mathrm{~Hz}$ stimulation, (Fig. 14C), and peak shifts during stimulation wash in and wash out (Fig. $14 E, F)$. These changes in the peak frequency of the gamma oscillation did not produce observable changes in the clinical phenomenology of the dyskinetic movements.

\section{Discussion}

We studied circuit mechanisms of dyskinesia using a novel, totally implanted chronic multisite brain-recording device in humans with PD. Motor cortex ECoG and STN LFPs were analyzed in two patients over 1 year, revealing a narrowband network oscillation between 60 and $90 \mathrm{~Hz}$. This oscillation is closely associated with dyskinesia. When dyskinesia is present during DBS, this oscillation is entrained at a subharmonic of the stimulation frequency. These findings provide a mechanis- 
Table 4. Gamma oscillation characteristics for motor cortex ECOG and STN LFP power spectra off stimulation

\begin{tabular}{llll}
\hline Measure & Mean peak frequency & Mean peak height & Mean peak width \\
\hline Motor cortex & $74.56 \mathrm{~Hz}(\mathrm{SD}, 3.59 \mathrm{~Hz})$ & $7.25(\mathrm{SD}, 3.01) 10 \times \log _{10}\left(\mu \mathrm{V}^{2} / \mathrm{Hz}\right)$ & $5.15 \mathrm{~Hz}(\mathrm{SD}, 1.28 \mathrm{~Hz})$ \\
STN & $72.95 \mathrm{~Hz}(\mathrm{SD}, 3.30 \mathrm{~Hz})$ & $3.01(\mathrm{SD}, 2.14) 10 \times \log _{10}\left(\mu \mathrm{V} /{ }^{2} \mathrm{~Hz}\right)$ & $4.37 \mathrm{~Hz}(\mathrm{SD}, 1.47 \mathrm{~Hz})$ \\
\hline
\end{tabular}

\section{A Frequencies of gamma oscillation Off DBS during dyskinesia}

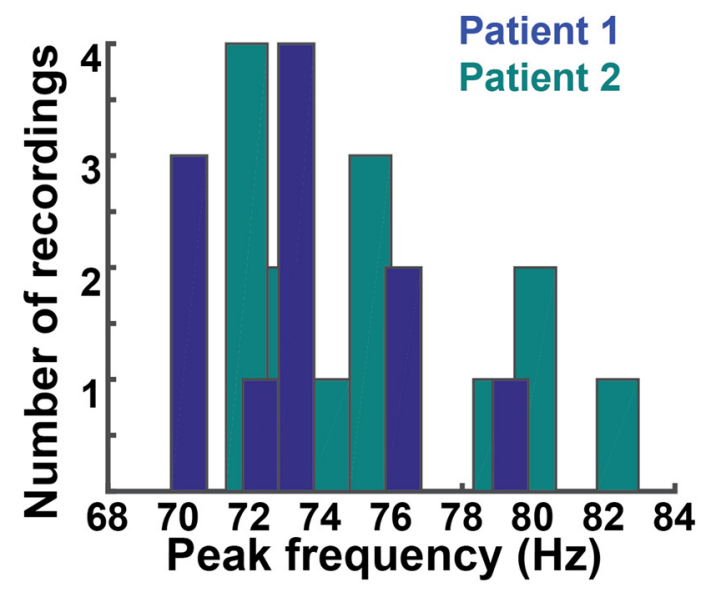

\section{B Correlations of frequency in motor cortex and STN during dyskinesia}

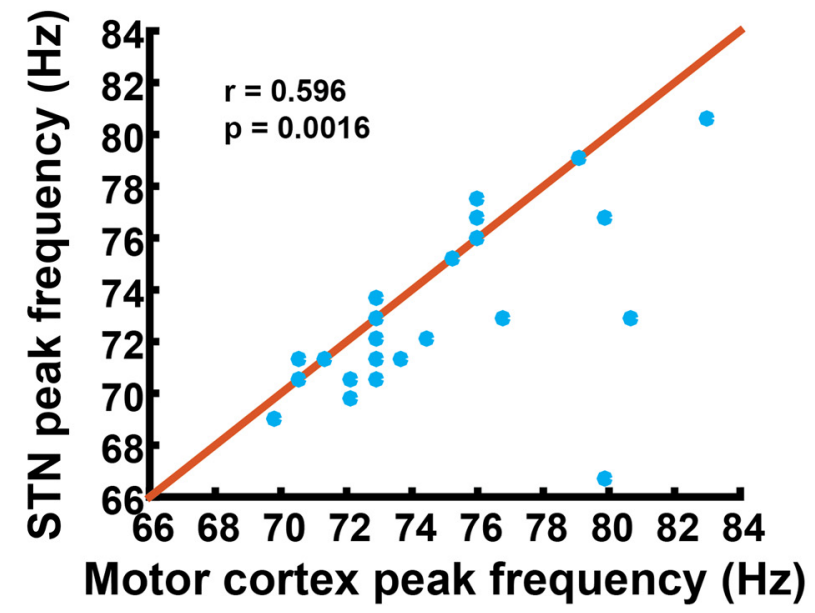

Figure 9. Characterization of peak frequency of the gamma oscillation. $A$, Frequencies at which the gamma oscillation occurred off DBS for Patients 1 and 2 . There does not appear to be a "characteristic frequency" within the gamma range for each patient. $\boldsymbol{B}$, Correlation between frequency of gamma oscillation in motor cortex versus STN for stimulation off files (Pearson's $r=0.597, p=0.0016)$. The $y=x$ line is shown in red.

tic basis for a debilitating adverse effect of therapy in PD and suggest algorithms for feedback-controlled neurostimulation.

Narrowband gamma rhythms vs broadband gamma activity The narrowband 60-90 Hz rhythm studied here should be distinguished from cortical "broadband gamma," which is a wideband phenomenon (typically 50-200 Hz) that tracks local activation and likely reflects underlying spiking activity rather than a narrowband oscillatory rhythm (Ray et al., 2008; Manning et al., 2009; Scheffer-Teixeira et al., 2013). Broadband gamma activity may play a role in the pathophysiology of the offmedication parkinsonian state, where akinesia is prominent and dyskinesia is absent. In this state, there is elevated resting broadband gamma in motor cortex (Crowell et al., 2012; Rowland et al., 2015) as well as excessive coupling of broadband gamma to the phase of the beta rhythm (de Hemptinne et al., 2013). Broadband gamma is also modulated by voluntary movement (Crone et al., 1998a; Miller et al., 2007). In contrast, the narrowband gamma oscillation elucidated in the present work is likely a manifestation of neuronal oscillations, is relatively unchanged by voluntary movements, and is associated with a hyperkinetic, rather than a bradykinetic, state. For illustration of this distinction see Figure 12. We use the terms "oscillation" or "rhythm" to make the distinction between narrowband phenomena versus arrhythmic broadband phenomena.

Functional role of gamma oscillations and relationship to hyperkinetic movements

Previous work has shown narrowband gamma oscillations to be a normal feature of cortical function, implicated in numerous cognitive processes, which may act to "bind" cortical regions together based on their phase relationships (Fries, 2009; Sohal, 2012). Alterations in cortical gamma rhythms have been implicated in diseases including autism and schizophrenia (Sohal, 2012). Altering the balance of excitatory and inhibitory activity in the cortex regulates these rhythms (Yizhar et al., 2011). Interactions of inhibitory fast-spiking interneurons play a critical role (Sohal, 2012; Salkoff et al., 2015), and these generators may be regulated by neuromodulators including acetylcholine (Teles-Grilo Ruivo and Mellor, 2013) and serotonin (Puig et al., 2010).

In subcortical regions, invasive human LFP recordings from temporarily externalized DBS leads have detected gamma rhythms in STN (Cassidy et al., 2002; Alonso-Frech et al., 2006; Trottenberg et al., 2006), globus pallidus (Weinberger et al., 2012), and thalamus in both PD patients and in patients with several nonparkinsonian conditions (Kempf et al., 2009). In PD patients, the subcortical gamma oscillation is present mainly in the on-medication state. However, the relationship to dyskinesia has been unclear. A combined STN LFP/magnetoencephalography study in PD detected a cortical gamma rhythm and STN-cortical coherence at the onset of voluntary movement, supporting a prokinetic role for the gamma oscillation (Litvak et al., 2012). Here, we are able to characterize a gamma oscillation that propagates through motor cortex and basal ganglia and is strongly associated with dyskinesia. Given the presence of gamma oscillations in the thalamus in several nondyskinetic conditions (Kempf et al., 2009), it is possible that dyskinesia arises when subcortical gamma rhythms are excessively propagated throughout the basal ganglia-thalamocortical loop, with a prominent representation in motor cortex. In support of this view, motor cortex recordings in a rodent model of parkinsonism showed a gamma oscillation during dyskinesia remarkably similar to that reported here (Halje et al., 2012). Together, these finding support the hypothesis that oscillations play a fundamental role in brain network dynamics and that alternations of these oscillations may manifest as disease (Voytek and Knight, 2015). 
A

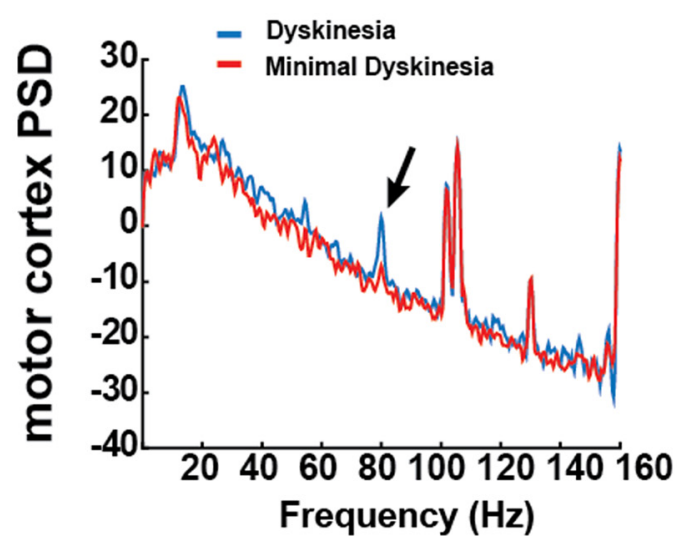

B

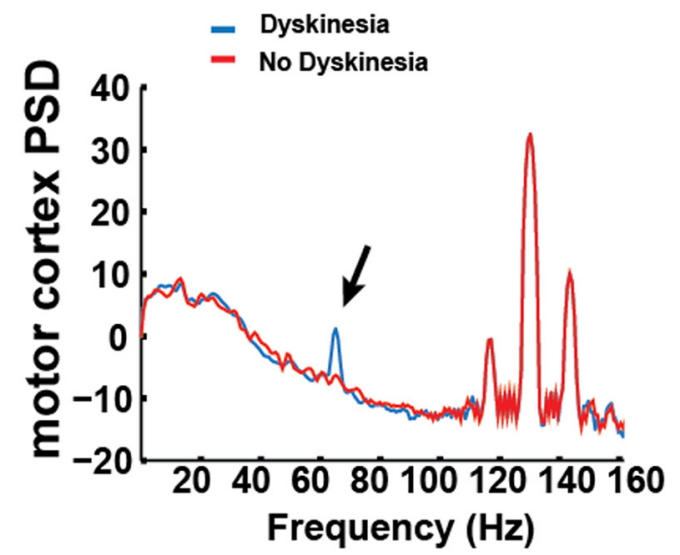

Figure 10. Example recordings from patients not included in the grouped statistical analysis. $\boldsymbol{A}, \boldsymbol{B}$, Examples from Patients $3(\boldsymbol{A})$ and $4(\boldsymbol{B})$ during episodes of dyskinesia versus no dyskinesia (or minimal dyskinesia). Both recordings are on DBS $(160 \mathrm{~Hz}$ for $\boldsymbol{A}, 130 \mathrm{~Hz}$ for $\boldsymbol{B})$. Note that in $\boldsymbol{A}$, the data were recorded at $422 \mathrm{~Hz}$ during a montage recording. In $\boldsymbol{B}$, data were recorded at $800 \mathrm{~Hz}$ as usual. The PSD scale is $10 * \log _{10}\left(\mu V^{2} / \mathrm{Hz}\right)$.

The mechanism by which gamma oscillations lead to dyskinesia is speculative. Oscillations can bias the probability of spike discharge, such that neuronal spiking tends to occur at a preferred oscillatory phase. Gamma oscillations tend to synchronize cortical neuronal pools so that common inputs to a cell arrive in close temporal succession, facilitating activation (Fries et al., 2001; Fries, 2009). In primary motor cortex, "fragments of movement" appear to be encoded by small groups of neurons, as demonstrated by the induction of complex movements by microstimulation (Graziano et al., 2002; Hatsopoulos et al., 2007). Thus, the coordination of these neuronal pools by an exaggerated gamma oscillation could release locally encoded fragments in rapid progression to produce choreiform activity.

Striatal mechanisms of levodopainduced dyskinesia: possible links to gamma oscillations

The cellular origin of levodopa-induced dyskinesia remains controversial, but most current theories, developed in rodent models, emphasize changes in striatal microcircuitry induced by dopamine denervation followed by its unregulated restoration by medication (Fieblinger and Cenci, 2015). Pulsatile dopamine release may be mediated by serotonergic neurons (Carta et al., 2007). Striatal changes include morphological alternations in glutamatergic synapses onto striatal medium spiny neurons and concomitant changes in long-term potentiation and long-term depression, which strengthen corticostriatal connections (Fieblinger and Cenci, 2015). These changes might favor activation of striatal cells originating the prokinetic "direct" intrinsic basal ganglia pathway (Thiele et al., 2014).

A challenge in this field is relating the changes in striatal physiology to oscillatory phenomenon. We and others have proposed
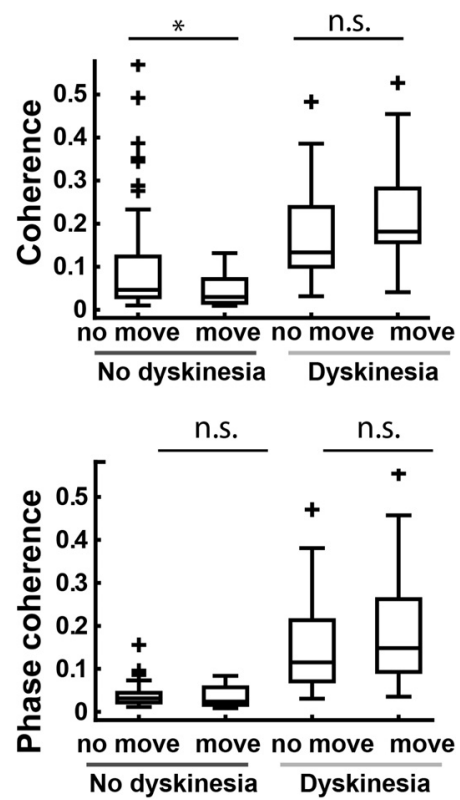

Figure 11. Amplitudes of dyskinesia biomarkers related to the gamma oscillation are minimally affected by voluntary movement. Biomarker amplitudes associated with each recording were segregated both by presence or absence of dyskinesia and presence or absence of voluntary movement (i.e., "walking" or "iPad" recordings compared to other recordings). There were 20 or more recordings included in the analysis for each condition. The data set is the same as that used in Figure 2C. Except for coherence in the nondyskinetic state ( $p=0.0063)$, the movement condition had no effect on biomarker amplitude. n.s., Not significant. Data are displayed with boxplots as in Figure $2 C$. ${ }^{*}$ indicates $p<0.01$.

that striatal changes in the parkinsonian off state have the effect of reducing the basal ganglia "filter" of cortical activity, such that normal rhythms, including the motor beta rhythm, are excessively transmitted through the basal ganglia-thalamocortical loop, resulting in aberrant beta synchronization (Weinberger and Dostrovsky, 2011; de Hemptinne et al., 2013). A similar mechanism could underlie dyskinesia, again due to exaggerated "propagation" of an otherwise normal physiological rhythm through abnormally strengthened corticostriatal synapses.

Entrainment of gamma oscillations by stimulation

Clinically, dyskinesia is not only associated with levodopa in PD, but may be induced by DBS in both PD (Yelnik et al., 2000) and 
A No Dyskinesia

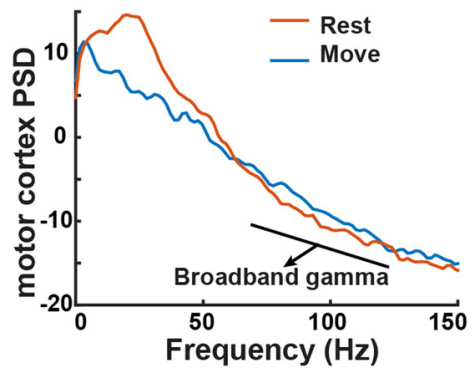

B Dyskinesia

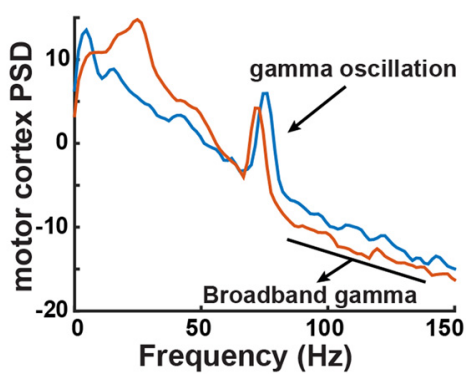

Figure 12. Broadband gamma versus gamma oscillation. $A, B$, Example of broadband gamma increase during movement when Patient 2 was not dyskinetic $(\boldsymbol{A})$ and when he was dyskinetic $(\boldsymbol{B})$. Note that in $\boldsymbol{B}$, the gamma oscillation is also apparent, and distinguishable from the broadband increase. Plots are derived from 20 trials of the iPad reaching task (analysis contains $2 \mathrm{~s}$ of movement, $2 \mathrm{~s}$ of rest for each trial). In $\boldsymbol{A}$, the patient was off medications, and in $\boldsymbol{B}$ they were on medications. In both examples they were off DBS. The PSD scale is $10 * \log _{10}\left(\mu \mathrm{V}^{2} / \mathrm{Hz}\right)$.

non-PD disorders (Mouton et al., 2006; Mallet et al., 2008; Ostrem et al., 2011). The entrainment of gamma rhythms by DBS (Fig. 14) offers a potential explanation for this. DBS is often delivered at $120-180 \mathrm{~Hz}$, approximately twice the typical frequency of the dyskinesia-associated gamma oscillation. DBS entrains axonal activity to stimulus pulses, but this entrainment does not occur after every pulse (Li et al., 2012). Frequent failures of entrainment (in this case, every other stimulation pulse) could readily result in driving axonal or neuronal activity at $60-90 \mathrm{~Hz}$. Our results suggest that irregular stimulation paradigms may be less prodyskinetic than the constant frequency stimulation that is used currently.
A

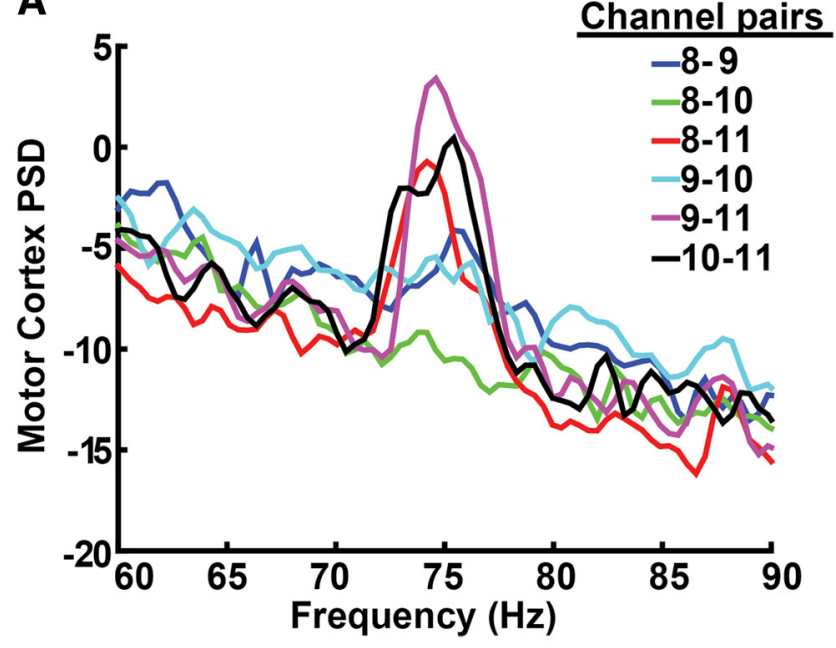

B

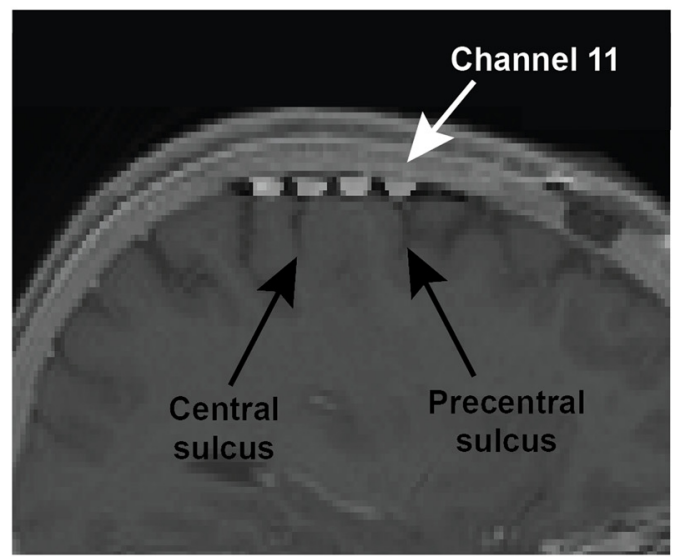

Figure 13. Optimal recording location for cortical gamma oscillations. $A$, Set of recordings from all contact pairs recorded sequentially (over a time period of $3 \mathrm{~min}$ ) from Patient 2 during dyskinesia. This recording was obtained with a sampling rate of $422 \mathrm{~Hz}$ (required to obtain a rapid sequential "montage recording" across all possible contact pairs) with DBS off. The peak is most strongly observed in contact pairs that include contact 11. The PSD scale is $10{ }^{*} \log _{10}\left(\mu \mathrm{V}^{2} / \mathrm{Hz}\right)$. B , Fusion of intraoperative (T with preoperative MRI, showing contact locations with respect to gyral anatomy. Contact 11 (white arrow) is located over the anterior portion of the precentral gyrus as well as precentral sulcus.

\section{Control signals for closed-loop DBS}

The finding of a brain rhythm reliably associated with dyskinesia has translational potential as a control signal in closed-loop DBS (Rosin et al., 2011; Little et al., 2013). Commercially available DBS systems are "open-loop" devices that do not respond to patients' symptom fluctuation. Stimulation-induced dyskinesia can be an important dose-limiting effect of DBS (Mouton et al., 2006; Mallet et al., 2008; Ostrem et al., 2011). To address this, a system could be designed that sensed the gamma rhythm and dynamically adjusted stimulation parameters to keep motor cortical spectral power in the relevant bandwidth below a specified level, mitigating hyperkinetic adverse effects. The cortical sensor provides a signal that is easily separable from normal cortical oscillatory activity and has less stimulation artifact than signals detected from the DBS lead. Control signals for closed-loop DBS related to akinesia, based on activity in the beta band, are under exploration (Little et al., 2013). However, beta activity is strongly affected by voluntary movement (Crone et al., 1998b; de Hemptinne et al., 2015), which may present a challenge for its use in closed-loop DBS.

\section{Limitations}

Determining the specificity of the gamma oscillation for dyskinesia depends on the accuracy of dyskinesia detection. Inclinic scoring was performed by a movement disorders neurologist, but the relative insensitivity of the scoring system and difficulty of continuously assessing dyskinesia during brain recording precluded precise examination of the timing of neural activity relative to changes in dyskinesia severity. Since dyskinesia is not always present during study visits, we chose to increase our number of recordings by allowing patients to trigger recordings at home. A cognitively intact PD patient with a history of dyskinesia is capable of scoring dyskinesia as present or absent, but we do not have independent verification of dyskinesia for home recordings. Additionally, because we used an implant without microrecording capability, our study focused on network rhythms recorded from macroelectrodes and did not address the relationship between dyskinesia and single unit discharge. However, it is likely that gamma oscillations exert a strong effect on neuronal discharge by entrainment to a preferred phase of the gamma rhythm (Trottenberg et al., 2006; Halje et al., 2012). 


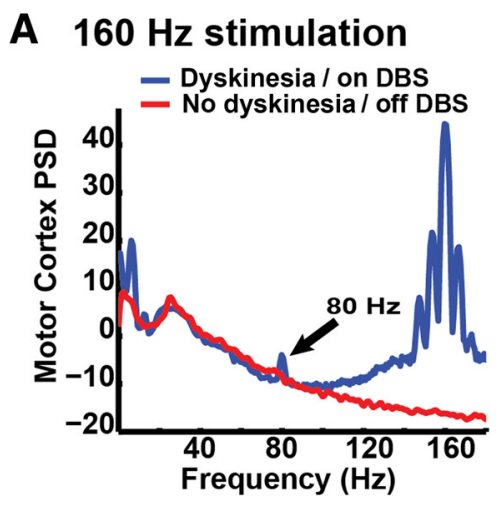

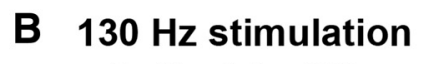

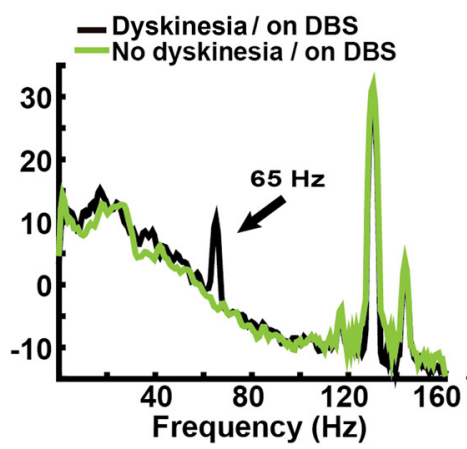

C Changing from $130 \mathrm{~Hz}$ stimulation to $150 \mathrm{~Hz}$

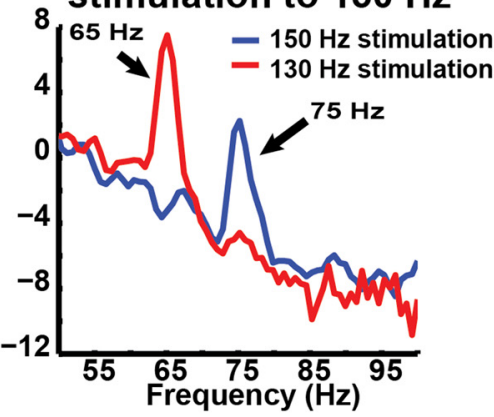

D Phase angle on DBS
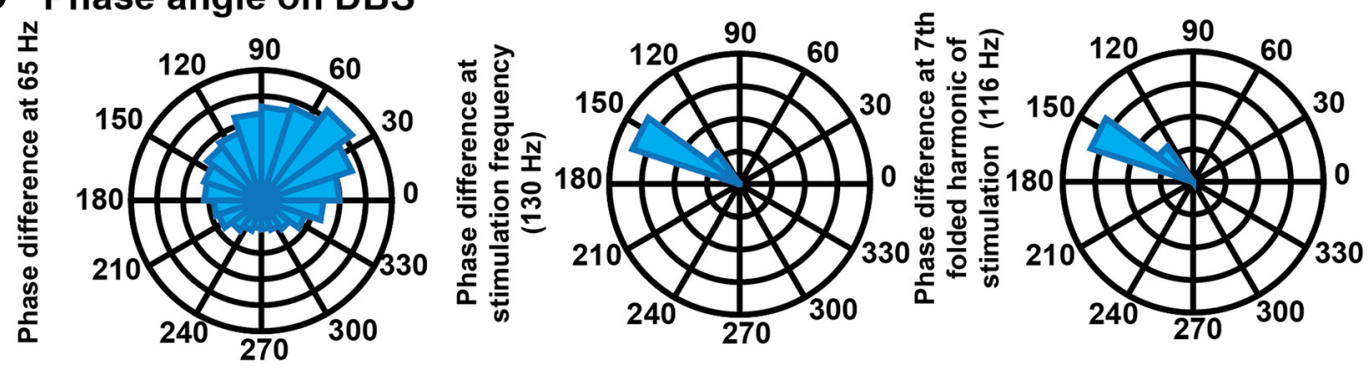

E Wash in
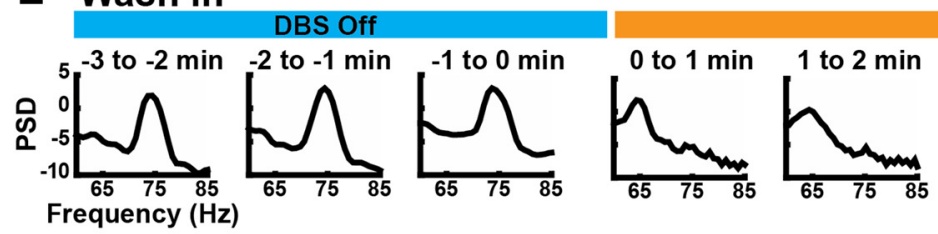

DBS On
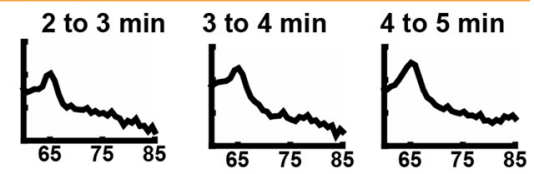

\section{F Wash out}
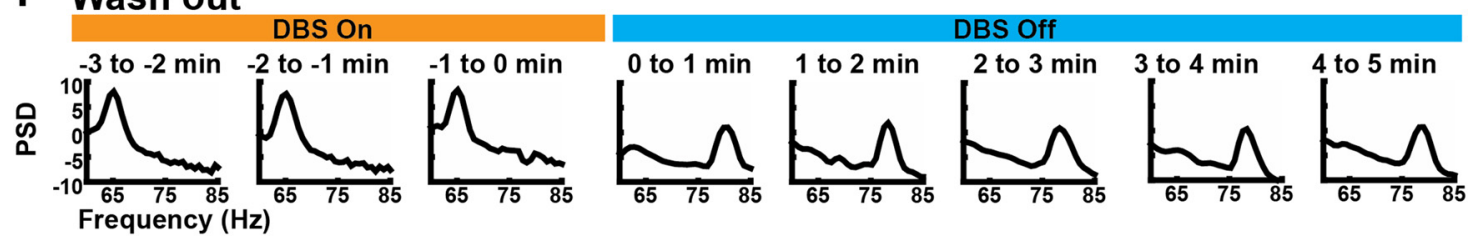

Figure 14. DBS entrains the gamma oscillation at half the stimulation frequency. A, Example from Patient 1 during dyskinesia that was present on DBS (160 Hz) and absent off DBS. The PSD scale is $10 * \log _{10}\left(\mu \mathrm{V}^{2} / \mathrm{Hz}\right)$. B, Example from Patient 2 with and without dyskinesia on DBS $(130 \mathrm{~Hz})$. Note that the gamma oscillation is not present in the recording without dyskinesia, despite a similar stimulation artifact, arguing against the artifact driving the effect. C, When DBS frequency is changed (from 130 to $150 \mathrm{~Hz}$ ), the gamma oscillation also changes (from 65 to $75 \mathrm{~Hz}$ ). An example from Patient 2 is shown. $\boldsymbol{D}$, Instantaneous phase difference between STN and motor cortex for a file recorded during DBS while the patient was experiencing dyskinesia. The gamma oscillation has a similar distribution and phase angle as the off stimulation recordings (compare Fig. $6 B$ ). In contrast, the same measures at the stimulation frequency $(130 \mathrm{~Hz})$ or a folded subharmonic (116 Hz) have a more narrow distribution, consistent with an artifactual source. Example from Patient 2. $E$, The frequency of the gamma oscillation during DBS wash-in moves from $\sim 75 \mathrm{~Hz}$ to half the stimulation frequency ( $65 \mathrm{~Hz}$, at $130 \mathrm{~Hz}$ stimulation). $\boldsymbol{F}$, During washout, the gamma oscillation moves back to $\sim 75-80 \mathrm{~Hz}$. Both $\boldsymbol{E}$ and $\boldsymbol{F}$ are from Patient 2 .

\section{Conclusions}

Using multisite chronic brain recordings in humans with PD, we characterize the association of a gamma oscillation in the basal ganglia-cortical motor network with dyskinesia. These findings illuminate the network dynamics underlying dyskinesia and suggest a strategy for feedback-controlled DBS.

\section{References}

Agnesi F, Muralidharan A, Baker KB, Vitek JL, Johnson MD (2015) Fidelity of frequency and phase entrainment of circuit-level spike activity during DBS. J Neurophysiol 114:825-834. CrossRef Medline

Alegre M, López-Azcárate J, Alonso-Frech F, Rodríguez-Oroz MC, Valencia M, Guridi J, Artieda J, Obeso JA (2012) Subthalamic activity during diphasic dyskinesias in Parkinson's disease. Mov Disord 27:1178-1181. CrossRef Medline

Alonso-Frech F, Zamarbide I, Alegre M, Rodríguez-Oroz MC, Guridi J, Manrique M, Valencia M, Artieda J, Obeso JA (2006) Slow oscillatory activity and levodopa-induced dyskinesias in Parkinson's disease. Brain 129: 1748-1757. CrossRef Medline

Bastide MF, Meissner WG, Picconi B, Fasano S, Fernagut PO, Feyder M, Francardo V, Alcacer C, Ding Y, Brambilla R, Fisone G, Jon Stoessl A, Bourdenx M, Engeln M, Navailles S, De Deurwaerdère P, Ko WK, Simola N, Morelli M, Groc L, et al. (2015) Pathophysiology of L-dopa-induced motor and non-motor complications in Parkinson's disease. Prog Neurobiol 132:96-168. CrossRef Medline

Brown P, Williams D (2005) Basal ganglia local field potential activity: character and functional significance in the human. Clin Neurophysiol 116: 2510-2519. CrossRef Medline

Cagnan H, Kuhn AA, Brown P (2014) Co-modulation of finely tuned highgamma band activity across hemispheres in Parkinson's disease. Clin Neurophysiol 125:777-785. CrossRef Medline

Carta M, Carlsson T, Kirik D, Björklund A (2007) Dopamine released from 5-HT terminals is the cause of L-DOPA-induced dyskinesia in parkinsonian rats. Brain 130:1819-1833. CrossRef Medline 
Cassidy M, Mazzone P, Oliviero A, Insola A, Tonali P, Di Lazzaro V, Brown P (2002) Movement-related changes in synchronization in the human basal ganglia. Brain 125:1235-1246. CrossRef Medline

Crone NE, Miglioretti DL, Gordon B, Lesser RP (1998a) Functional mapping of human sensorimotor cortex with electrocorticographic spectral analysis. II. Event-related synchronization in the gamma band. Brain 121: 2301-2315. CrossRef Medline

Crone NE, Miglioretti DL, Gordon B, Sieracki JM, Wilson MT, Uematsu S, Lesser RP (1998b) Functional mapping of human sensorimotor cortex with electrocorticographic spectral analysis. I. Alpha and beta eventrelated desynchronization. Brain 121:2271-2299. CrossRef Medline

Crowell AL, Ryapolova-Webb ES, Ostrem JL, Galifianakis NB, Shimamoto S, Lim DA, Starr PA (2012) Oscillations in sensorimotor cortex in movement disorders: an electrocorticography study. Brain 135:615-630. CrossRef Medline

de Hemptinne C, Ryapolova-Webb ES, Air EL, Garcia PA, Miller KJ, Ojemann JG, Ostrem JL, Galifianakis NB, Starr PA (2013) Exaggerated phase-amplitude coupling in the primary motor cortex in Parkinson disease. Proc Natl Acad Sci U S A 110:4780-4785. CrossRef Medline

de Hemptinne C, Swann NC, Ostrem JL, Ryapolova-Webb ES, San Luciano M, Galifianakis NB, Starr PA (2015) Therapeutic deep brain stimulation reduces cortical phase-amplitude coupling in Parkinson's disease. Nat Neurosci 18:779-786. CrossRef Medline

Delorme A, Makeig S (2004) EEGLAB: an open source toolbox for analysis of single-trial EEG dynamics including independent component analysis. J Neurosci Methods 134:9-21. CrossRef Medline

Fieblinger T, Cenci MA (2015) Zooming in on the small: the plasticity of striatal dendritic spines in L-DOPA-induced dyskinesia. Mov Disord 30: 484-493. CrossRef Medline

Fries P (2005) A mechanism for cognitive dynamics: neuronal communication through neuronal coherence. Trends Cogn Sci 9:474-480. CrossRef Medline

Fries P (2009) Neuronal gamma-band synchronization as a fundamental process in cortical computation. Annu Rev Neurosci 32:209-224. CrossRef Medline

Fries P, Reynolds JH, Rorie AE, Desimone R (2001) Modulation of oscillatory neuronal synchronization by selective visual attention. Science 291: 1560-1563. CrossRef Medline

Garcia L, Audin J, D’Alessandro G, Bioulac B, Hammond C (2003) Dual effect of high-frequency stimulation on subthalamic neuron activity. J Neurosci 23:8743-8751. Medline

Goetz CG, Nutt JG, Stebbins GT (2008) The Unified Dyskinesia Rating Scale: presentation and clinimetric profile. Mov Disord 23:2398-2403. CrossRef Medline

Graziano MS, Taylor CS, Moore T (2002) Complex movements evoked by microstimulation of precentral cortex. Neuron 34:841-851. CrossRef Medline

Halje P, Tamtè M, Richter U, Mohammed M, Cenci MA, Petersson P (2012) Levodopa-induced dyskinesia is strongly associated with resonant cortical oscillations. J Neurosci 32:16541-16551. CrossRef Medline

Hammond C, Bergman H, Brown P (2007) Pathological synchronization in Parkinson's disease: networks, models and treatments. Trends Neurosci 30:357-364. CrossRef Medline

Hashimoto T, Elder CM, Okun MS, Patrick SK, Vitek JL (2003) Stimulation of the subthalamic nucleus changes the firing pattern of pallidal neurons. J Neurosci 23:1916-1923. Medline

Hatsopoulos NG, Xu Q, Amit Y (2007) Encoding of movement fragments in the motor cortex. J Neurosci 27:5105-5114. CrossRef Medline

Kempf F, Brücke C, Salih F, Trottenberg T, Kupsch A, Schneider GH, Doyle Gaynor LM, Hoffmann KT, Vesper J, Wöhrle J, Altenmuller DM, Krauss JK, Mazzone P, Di Lazzaro V, Yelnik J, Kühn AA, Brown P (2009) Gamma activity and reactivity in human thalamic local field potentials. Eur J Neurosci 29:943-953. CrossRef Medline

Kühn AA, Kupsch A, Schneider GH, Brown P (2006) Reduction in subthalamic $8-35 \mathrm{~Hz}$ oscillatory activity correlates with clinical improvement in Parkinson's disease. Eur J Neurosci 23:1956-1960. CrossRef Medline

Kühn AA, Kempf F, Brücke C, Gaynor Doyle L, Martinez-Torres I, Pogosyan A, Trottenberg T, Kupsch A, Schneider GH, Hariz MI, Vandenberghe W, Nuttin B, Brown P (2008) High-frequency stimulation of the subthalamic nucleus suppresses oscillatory beta activity in patients with Parkinson's disease in parallel with improvement in motor performance. J Neurosci 28:6165-6173. CrossRef Medline
Li Q, Ke Y, Chan DC, Qian ZM, Yung KK, Ko H, Arbuthnott GW, Yung WH (2012) Therapeutic deep brain stimulation in Parkinsonian rats directly influences motor cortex. Neuron 76:1030-1041. CrossRef Medline

Little S, Pogosyan A, Neal S, Zavala B, Zrinzo L, Hariz M, Foltynie T, Limousin P, Ashkan K, FitzGerald J, Green AL, Aziz TZ, Brown P (2013) Adaptive deep brain stimulation in advanced Parkinson disease. Ann Neurol 74:449-457. CrossRef Medline

Litvak V, Eusebio A, Jha A, Oostenveld R, Barnes G, Foltynie T, Limousin P, Zrinzo L, Hariz MI, Friston K, Brown P (2012) Movement-related changes in local and long-range synchronization in Parkinson's disease revealed by simultaneous magnetoencephalography and intracranial recordings. J Neurosci 32:10541-10553. CrossRef Medline

Mallet L, Polosan M, Jaafari N, Baup N, Welter ML, Fontaine D, du Montcel ST, Yelnik J, Chéreau I, Arbus C, Raoul S, Aouizerate B, Damier P, Chabardès S, Czernecki V, Ardouin C, Krebs MO, Bardinet E, Chaynes $\mathrm{P}$, Burbaud P, et al. (2008) Subthalamic nucleus stimulation in severe obsessive-compulsive disorder. N Engl J Med 359:2121-2134. CrossRef Medline

Manning JR, Jacobs J, Fried I, Kahana MJ (2009) Broadband shifts in local field potential power spectra are correlated with single-neuron spiking in humans. J Neurosci 29:13613-13620. CrossRef Medline

Miller KJ, Leuthardt EC, Schalk G, Rao RP, Anderson NR, Moran DW, Miller JW, Ojemann JG (2007) Spectral Changes in Cortical Surface Potentials during Motor Movement. J Neurosci 27:2424-2432. CrossRef Medline

Moran A, Bergman H, Israel Z, Bar-Gad I (2008) Subthalamic nucleus functional organization revealed by parkinsonian neuronal oscillations and synchrony. Brain 131:3395-3409. CrossRef Medline

Mouton S, Xie-Brustolin J, Mertens P, Polo G, Damier P, Broussolle E, Thobois S (2006) Chorea induced by globus pallidus externus stimulation in a dystonic patient. Mov Disord 21:1771-1773. CrossRef Medline

Ostrem JL, Racine CA, Glass GA, Grace JK, Volz MM, Heath SL, Starr PA (2011) Subthalamic nucleus deep brain stimulation in primary cervical dystonia. Neurology 76:870-878. CrossRef Medline

Oyama G, Foote KD, Jacobson CE 4th, Velez-Lago F, Go C, Limotai N, Zeilman PR, Romrell J, Wu SS, Neal D, Okun MS (2012) GPi and STN deep brain stimulation can suppress dyskinesia in Parkinson's disease. Parkinsonism Relat Disord 18:814-818. CrossRef Medline

Priori A, Foffani G, Pesenti A, Tamma F, Bianchi AM, Pellegrini M, Locatelli M, Moxon KA, Villani RM (2004) Rhythm-specific pharmacological modulation of subthalamic activity in Parkinson's disease. Exp Neurol 189:369-379. CrossRef Medline

Pritchard WS (1992) The brain in fractal time: 1/f-like power spectrum scaling of the human electroencephalogram. Int J Neurosci 66:119-129. CrossRef Medline

Puig MV, Watakabe A, Ushimaru M, Yamamori T, Kawaguchi Y (2010) Serotonin modulates fast-spiking interneuron and synchronous activity in the rat prefrontal cortex through 5-HT1A and 5-HT2A receptors. J Neurosci 30:2211-2222. CrossRef Medline

Ray S, Crone NE, Niebur E, Franaszczuk PJ, Hsiao SS (2008) Neural correlates of high-gamma oscillations $(60-200 \mathrm{~Hz})$ in macaque local field potentials and their potential implications in electrocorticography. J Neurosci 28:11526-11536. CrossRef Medline

Rosin B, Slovik M, Mitelman R, Rivlin-Etzion M, Haber SN, Israel Z, Vaadia E, Bergman H (2011) Closed-loop deep brain stimulation is Superior in ameliorating parkinsonism. Neuron 72:370-384. CrossRef Medline

Rowland NC, De Hemptinne C, Swann NC, Qasim S, Miocinovic S, Ostrem JL, Knight RT, Starr PA (2015) Task-related activity in sensorimotor cortex in Parkinson's disease and essential tremor: changes in beta and gamma bands. Front Hum Neurosci 9:512. Medline

Salkoff DB, Zagha E, Yüzgeç Ö, McCormick DA (2015) Synaptic mechanisms of tight spike synchrony at gamma frequency in cerebral cortex. J Neurosci 35:10236-10251. CrossRef Medline

Scheffer-Teixeira R, Belchior H, Leão RN, Ribeiro S, Tort AB (2013) On high-frequency field oscillations $(>100 \mathrm{~Hz})$ and the spectral leakage of spiking activity. J Neurosci 33:1535-1539. CrossRef Medline

Shahlaie K, Larson PS, Starr PA (2011) Intraoperative computed tomography for deep brain stimulation surgery: technique and accuracy assessment. Neurosurgery 68:114-124; discussion 124. Medline

Siegel M, Donner TH, Engel AK (2012) Spectral fingerprints of large-scale neuronal interactions. Nat Rev Neurosci 13:121-134. Medline

Silberstein P, Pogosyan A, Kühn AA, Hotton G, Tisch S, Kupsch A, DowseyLimousin P, Hariz MI, Brown P (2005) Cortico-cortical coupling in 
Parkinson's disease and its modulation by therapy. Brain 128:1277-1291. CrossRef Medline

Sohal VS (2012) Insights into cortical oscillations arising from optogenetic studies. Biol Psychiatry 71:1039-1045. CrossRef Medline

Starr PA, Christine CW, Theodosopoulos PV, Lindsey N, Byrd D, Mosley A, Marks WJ Jr (2002) Implantation of deep brain stimulators into the subthalamic nucleus: technical approach and magnetic resonance imaging-verified lead locations. J Neurosurg 97:370-387. CrossRef Medline

Teles-Grilo Ruivo LM, Mellor JR (2013) Cholinergic modulation of hippocampal network function. Front Synaptic Neurosci 5:2. Medline

Thiele SL, Chen B, Lo C, Gertler TS, Warre R, Surmeier JD, Brotchie JM, Nash JE (2014) Selective loss of bi-directional synaptic plasticity in the direct and indirect striatal output pathways accompanies generation of parkinsonism and l-DOPA induced dyskinesia in mouse models. Neurobiol Dis 71:334-344. CrossRef Medline

Trottenberg T, Fogelson N, Kühn AA, Kivi A, Kupsch A, Schneider GH, Brown P (2006) Subthalamic gamma activity in patients with Parkinson's disease. Exp Neurol 200:56-65. CrossRef Medline

Voytek B, Knight RT (2015) Dynamic network communication as a unifying neural basis for cognition, development, aging, and disease. Biol Psychiatry 77:1089-1097. CrossRef Medline

Weinberger M, Dostrovsky JO (2011) A basis for the pathological oscillations in basal ganglia: the crucial role of dopamine. Neuroreport 22: 151-156. CrossRef Medline

Weinberger M, Hutchison WD, Alavi M, Hodaie M, Lozano AM, Moro E, Dostrovsky JO (2012) Oscillatory activity in the globus pallidus inter- nus: comparison between Parkinson's disease and dystonia. Clin Neurophysiol 123:358-368. CrossRef Medline

Williams D, Tijssen M, Van Bruggen G, Bosch A, Insola A, Di Lazzaro V, Mazzone P, Oliviero A, Quartarone A, Speelman H, Brown P (2002) Dopamine-dependent changes in the functional connectivity between basal ganglia and cerebral cortex in humans. Brain 125:1558-1569. CrossRef Medline

Yelnik J, Damier P, Bejjani BP, Francois C, Gervais D, Dormont D, Arnulf I, M Bonnet A, Cornu P, Pidoux B, Agid Y (2000) Functional mapping of the human globus pallidus: contrasting effect of stimulation in the internal and external pallidum in Parkinson's disease. Neuroscience 101: 77-87. CrossRef Medline

Yizhar O, Fenno LE, Prigge M, Schneider F, Davidson TJ, O’Shea DJ, Sohal VS, Goshen I, Finkelstein J, Paz JT, Stehfest K, Fudim R, Ramakrishnan C, Huguenard JR, Hegemann P, Deisseroth K (2011) Neocortical excitation/inhibition balance in information processing and social dysfunction. Nature 477:171-178. CrossRef Medline

Yousry TA, Schmid UD, Alkadhi H, Schmidt D, Peraud A, Buettner A, Winkler P (1997) Localization of the motor hand area to a knob on the precentral gyrus. A new landmark. Brain 120:141-157. CrossRef Medline

Zheng Z, Li Y, Li J, Zhang Y, Zhang X, Zhuang P (2010) Stimulationinduced dyskinesia in the early stage after subthalamic deep brain stimulation. Stereotact Funct Neurosurg 88:29-34. CrossRef Medline

Zwieg MH, Campbell G (1993) Receiver-operating characteristic (ROC) plots: a fundamental evaluation tool in clinical medicine. Clin Chem 39:561-577. Medline 